\title{
Ground state solutions of the non-autonomous Schrödinger-Bopp-Podolsky system
}

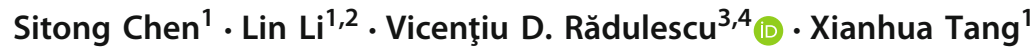

Received: 25 August 2021 / Revised: 26 August 2021 / Accepted: 30 August 2021 /

Published online: 10 December 2021

(C) The Author(s) 2021

\section{Abstract}

In this paper, we consider the following non-autonomous Schrödinger-BoppPodolsky system

$$
\left\{\begin{array}{l}
-\Delta u+V(x) u+q^{2} \phi u=f(u) \\
-\Delta \phi+a^{2} \Delta^{2} \phi=4 \pi u^{2}
\end{array} \text { in } \mathbb{R}^{3} .\right.
$$

By using some original analytic techniques and new estimates of the ground state energy, we prove that this system admits a ground state solution under mild assumptions on $V$ and $f$. In the final part of this paper, we give a min-max characterization of the ground state energy.

Keywords Schrödinger-Bopp-Podolsky system · Ground state solution · Least energy squeeze method · Nehari-Pohožaev manifold · Concentration-compactness

$\bowtie$ Vicenţiu D. Rădulescu

radulescu@inf.ucv.ro

Sitong Chen

mathsitongchen@mail.csu.edu.cn

Lin Li

lilin420@gmail.com

Xianhua Tang

tangxh@mail.csu.edu.cn

1 School of Mathematics and Statistics, HNP-LAMA, Central South University, Changsha 410083, Hunan, People's Republic of China

2 School of Mathematics and Statistics, Chongqing Technology and Business University, Chongqing 400067, People's Republic of China

3 Faculty of Applied Mathematics, AGH University of Science and Technology, al. Mickiewicza 30, Kraków 30-059, Poland

4 Department of Mathematics, University of Craiova, Street A.I. Cuza 13, 200585 Craiova, Romania 
Mathematics Subject Classification 35J48 $\cdot$ 35J50 $\cdot$ 35Q60 $\cdot 47 \mathrm{~J} 30 \cdot 58 \mathrm{E} 50$

\section{Introduction}

Consider the following Schrödinger-Bopp-Podolsky system

$$
\left\{\begin{array}{l}
-\Delta u+V(x) u+q^{2} \phi u=f(u) \\
-\Delta \phi+a^{2} \Delta^{2} \phi=4 \pi u^{2}
\end{array} \quad \text { in } \mathbb{R}^{3},\right.
$$

where $u, \phi: \mathbb{R}^{3} \rightarrow \mathbb{R}, \omega, a>0, q \neq 0$.

This nonlinear system appears when we couple a Schrödinger field $\psi=\psi(t, x)$ with its electromagnetic field in the Bopp-Podolsky electromagnetic theory, and, in particular, in the electrostatic case for standing waves $\psi(t, x)=e^{i \omega t} u(x)$.

System (1.1) has a strong physical meaning especially in the Bopp-Podolsky theory, developed independently by Bopp [3] and Podolsky [24]. The Bopp-Podolsky theory is a second order gauge theory for the electromagnetic field. As the Mie theory [22] and its generalizations given by Born and Infeld [4-7], it was introduced to solve the "infinity problem", which appears in the classical Maxwell theory. In fact, by the well-known Gauss law (or Poisson equation), the electrostatic potential $\phi$ for a given charge distribution whose density is $\rho$ satisfies the equation

$$
-\Delta \phi=\rho \quad \text { in } \mathbb{R}^{3} \text {. }
$$

If $\rho=4 \pi \delta_{x_{0}}$, with $x_{0} \in \mathbb{R}^{3}$, the fundamental solution of (1.2) is $\mathcal{G}\left(x-x_{0}\right)$, where

$$
\mathcal{G}(x)=\frac{1}{|x|},
$$

and the electrostatic energy is

$$
\mathcal{E}_{\mathrm{M}}(\mathcal{G})=\frac{1}{2} \int_{\mathbb{R}^{3}}|\nabla \mathcal{G}|^{2}=+\infty
$$

Thus, Eq. (1.2) is replaced by

$$
-\operatorname{div}\left(\frac{\nabla \phi}{\sqrt{1-|\nabla \phi|^{2}}}\right)=\rho \quad \text { in } \mathbb{R}^{3}
$$

in the Born-Infeld theory and by

$$
-\Delta \phi+a^{2} \Delta^{2} \phi=\rho \quad \text { in } \mathbb{R}^{3}
$$

in the Bopp-Podolsky theory. In both cases, if $\rho=4 \pi \delta_{x_{0}}$, we are able to write explicitly the solutions of the respective equations and to see that their energy is finite. 
In particular, when we consider the differential operator $-\Delta+a^{2} \Delta^{2}$, we have that $\mathcal{K}\left(x-x_{0}\right)$, with

$$
\mathcal{K}(x):=\frac{1-e^{-|x| / a}}{|x|},
$$

is the fundamental solution of the equation

$$
-\Delta \phi+a^{2} \Delta^{2} \phi=4 \pi \delta_{x_{0}}
$$

Then $\mathcal{K}$ has no singularity in $x_{0}$ since it satisfies

$$
\lim _{x \rightarrow x_{0}} \mathcal{K}\left(x-x_{0}\right)=\frac{1}{a},
$$

and its energy is

$$
\mathcal{E}_{\mathrm{BP}}(\mathcal{K})=\frac{1}{2} \int_{\mathbb{R}^{3}}|\nabla \mathcal{K}|^{2}+\frac{a^{2}}{2} \int_{\mathbb{R}^{3}}|\Delta \mathcal{K}|^{2}<+\infty .
$$

Moreover, the Bopp-Podolsky theory may be interpreted as an effective theory for short distances (see [20]), while for large distances it is experimentally indistinguishable from the Maxwell theory. Thus, the Bopp-Podolsky parameter $a>0$, which has dimension of the inverse of mass, can be interpreted as a cut-off distance or can be linked to an effective radius for the electron. For more physical details we refer the reader to the recent papers $[1,2,9,10,16,17]$ and to references therein.

The differential operator $-\Delta+\Delta^{2}$ appears in various different interesting mathematical and physical situations; see [19] and the references therein.

Before stating our results, few preliminaries are in order. We introduce here the space $\mathcal{D}$ as the completion of $\mathcal{C}_{c}^{\infty}\left(\mathbb{R}^{3}\right)$ with respect to the norm $\sqrt{\|\nabla \phi\|_{2}^{2}+a^{2}\|\Delta \phi\|_{2}^{2}}$; see Sect. 2 for more properties on this space.

For fixed $a>0$ and $q \neq 0$, we say that a pair $(u, \phi) \in H^{1}\left(\mathbb{R}^{3}\right) \times \mathcal{D}$ is a solution of problem (1.1) if

$$
\begin{gathered}
\int_{\mathbb{R}^{3}}[\nabla u \nabla v+V(x) u v] \mathrm{d} x+q^{2} \int_{\mathbb{R}^{3}} \phi u v \mathrm{~d} x=\int_{\mathbb{R}^{3}} f(u) v \mathrm{~d} x, \quad \forall v \in H^{1}\left(\mathbb{R}^{3}\right), \\
\int_{\mathbb{R}^{3}} \nabla \phi \nabla \xi \mathrm{d} x+a^{2} \int_{\mathbb{R}^{3}} \Delta \phi \Delta \xi \mathrm{d} x=4 \pi \int_{\mathbb{R}^{3}} \phi u^{2} \mathrm{~d} x, \quad \forall \xi \in \mathcal{D} .
\end{gathered}
$$

We say that a solution $(u, \phi)$ is nontrivial whenever $u \not \equiv 0$; a solution is called a ground state solution if its energy is minimal among all nontrivial solutions. As described in Sect. 2, to solve problem (1.1) is equivalent to solving

$$
-\Delta u+V(x) u+q^{2}\left(\frac{1-e^{-|x| / a}}{|x|} * u^{2}\right) u=f(u) \text { in } \mathbb{R}^{3},
$$


whose solutions correspond to critical points of the energy functional defined in $H^{1}\left(\mathbb{R}^{3}\right)$ by

$$
\mathcal{I}(u)=\frac{1}{2} \int_{\mathbb{R}^{3}}\left[|\nabla u|^{2}+V(x) u^{2}\right] \mathrm{d} x+\frac{q^{2}}{4} \int_{\mathbb{R}^{3}}\left(\frac{1-e^{-|x| / a}}{|x|} * u^{2}\right) u^{2} \mathrm{~d} x-\int_{\mathbb{R}^{3}} F(u) \mathrm{d} x,
$$

where $F(u)=\int_{0}^{u} f(t) \mathrm{d} t$. A solution is called a ground state solution if its energy is minimal among all nontrivial solutions.

In this paper, we also consider the following "limit" system with a general nonlinearity $f$

$$
\left\{\begin{array}{l}
-\Delta u+V_{\infty} u+q^{2} \phi u=f(u) \\
-\Delta \phi+a^{2} \Delta^{2} \phi=4 \pi u^{2}
\end{array} \quad \text { in } \mathbb{R}^{3} .\right.
$$

To the best of our knowledge, there is no result on the existence of ground state solutions for systems (1.1) and (1.5). Inspired by [11,12,14,25], we will seek a ground state solution of Nehari-Pohožaev type for systems (1.1) and (1.5).

To state our results, we introduce the following assumptions:

(V1) $V \in \mathcal{C}\left(\mathbb{R}^{3},[0, \infty)\right)$ and $V_{\infty}:=\lim _{|y| \rightarrow \infty} V(y)=\sup _{x \in \mathbb{R}^{3}} V(x)>0$;

(V2) $V \in \mathcal{C}^{1}\left(\mathbb{R}^{3}, \mathbb{R}\right), \nabla V(x) \cdot x \in L^{\infty}\left(\mathbb{R}^{3}\right), 2 V(x)+\nabla V(x) \cdot x \geq 0$ and $\liminf _{|x| \rightarrow \infty}[2 V(x)+\nabla V(x) \cdot x]>0$

(F1) $f \in \mathcal{C}(\mathbb{R}, \mathbb{R})$, and there exist constants $\mathcal{C}>0$ and $p \in(2,6)$ such that

$$
|f(t)| \leq \mathcal{C}\left(1+|t|^{p-1}\right), \quad \forall t \in \mathbb{R}
$$

(F2) $f(t)=o(t)$ as $t \rightarrow 0$;

(F3) $F(t) \geq 0$ for all $t \in \mathbb{R}$ and $\lim _{|t| \rightarrow \infty} \frac{F(t)}{|t|^{3}}=\infty$;

(F4) the function $\frac{2 f(t) t-3 F(t)}{t^{3}}$ is nondecreasing on $(-\infty, 0)$ and $(0,+\infty)$.

Our first result is as follows.

Theorem 1.1 Assume that (V1), (V2) and (F1)-(F4) hold. Then problem (1.1) admits a ground state solution.

Remark 1.2 There are many functions which satisfy (V1) and (V2). An example is given by $V(x)=1-\frac{\sin ^{2}|x|}{1+|x|}$.

For the constant potential case, we replace the monotonicity condition (F4) with the super-quadratic condition which is easier to verify:

(F5) $f(t) t \geq 3 F(t)$ for all $t \in \mathbb{R}$, and there exist $\kappa>3 / 2$ and $r_{0}, \mathcal{C}_{0}>0$ such that

$$
\left|\frac{f(t)}{t}\right|^{\kappa} \leq \mathcal{C}_{0}[f(t) t-3 F(t)], \quad \forall|t| \geq r_{0}
$$


Our second result is as follows.

Theorem 1.3 Assume that (F1)-(F3) and (F5) hold. Then problem (1.5) admits a ground state solution.

Finally, we give the min-max property of the ground state energy of $\mathcal{I}$. To this end, we introduce the following monotonicity condition.

(V3) $V \in \mathcal{C}^{1}\left(\mathbb{R}^{3}\right)$, and the function $t \mapsto t^{2}[V(t x)-\nabla V(t x) \cdot(t x)]$ is increasing on $(0,+\infty)$ for every $x \in \mathbb{R}^{3}$.

We define the Nehari-Pohožaev manifold as follows:

$$
\mathcal{M}=\left\{u \in H^{1}\left(\mathbb{R}^{3}\right) \backslash\{0\}: \mathcal{J}(u):=2 \mathcal{I}^{\prime}(u)[u]-\mathcal{P}(u)=0\right\}
$$

where $\mathcal{P}(u)$ is the Pohožaev functional of (1.3) defined by

$$
\begin{aligned}
\mathcal{P}(u):= & \frac{1}{2}\|\nabla u\|_{2}^{2}+\frac{1}{2} \int_{\mathbb{R}^{3}}[3 V(x)+\nabla V(x) \cdot x] u^{2} \mathrm{~d} x-3 \int_{\mathbb{R}^{3}} F(u) \mathrm{d} x \\
& +\frac{q^{2}}{4 a} \int_{\mathbb{R}^{3}} \int_{\mathbb{R}^{3}}\left[5 \frac{1-e^{-\frac{|x-y|}{a}}}{|x-y| / a}+e^{-\frac{|x-y|}{a}}\right] u^{2}(x) u^{2}(y) \mathrm{d} x \mathrm{~d} y .
\end{aligned}
$$

If $u \in H^{1}\left(\mathbb{R}^{3}\right)$ is a critical point of $\mathcal{I}$, then $u$ satisfies $\mathcal{P}(u)=0$; see [18, A.14] for more details. Then every nontrivial solution of (1.1) is contained in $\mathcal{M}$. In this direction, we have the following theorem.

Theorem 1.4 Assume that (V1), (V3), (F1)-(F4) hold. Then problem (1.1) admits a ground state solution $\bar{u} \in H^{1}\left(\mathbb{R}^{3}\right)$ such that

$$
\mathcal{I}(\bar{u})=\inf _{\mathcal{M}} \mathcal{I}=\inf _{u \in H^{1}\left(\mathbb{R}^{3}\right) \backslash\{0\}} \max _{t>0} \mathcal{I}\left(t^{2} u_{t}\right)>0
$$

where $u_{t}(x):=u(t x)$.

Remark 1.5 We observe that the function $V(x)=1-\frac{1}{(1+|x|)^{\alpha}}$ with $\alpha>0$ satisfies hypotheses (V1) and (V3).

For the limiting problem related to (1.3), that is, (1.3) with $V(x) \equiv V_{\infty}$, we further weaken $(\mathrm{F} 4)$ to the following condition:

$\left(\mathrm{F} 4^{\prime}\right)$ there exists a constant $\theta \in[0,1)$ such that the function $\frac{4 f(t) t-6 F(t)-\theta V_{\infty} t}{2 t^{3}}$ is nondecreasing on $(-\infty, 0)$ and $(0,+\infty)$.

To state the following result, we define the energy functional in $H^{1}\left(\mathbb{R}^{3}\right)$ by

$$
\mathcal{I}^{\infty}(u)=\frac{1}{2} \int_{\mathbb{R}^{3}}\left[|\nabla u|^{2}+V_{\infty} u^{2}\right] \mathrm{d} x+\frac{q^{2}}{4} \int_{\mathbb{R}^{3}}\left(\frac{1-e^{-|x| / a}}{|x|} * u^{2}\right) u^{2} \mathrm{~d} x-\int_{\mathbb{R}^{3}} F(u) \mathrm{d} x,
$$


and the Nehari-Pohožaev manifold by

$$
\mathcal{M}^{\infty}:=\left\{u \in H^{1}\left(\mathbb{R}^{3}\right) \backslash\{0\}: \mathcal{J}^{\infty}(u):=2\left(\mathcal{I}^{\infty}\right)^{\prime}(u)[u]-\mathcal{P}^{\infty}(u)=0\right\},
$$

where $\mathcal{P}^{\infty}(u)$ is the Pohožaev functional defined by

$$
\begin{aligned}
\mathcal{P}^{\infty}(u):= & \frac{1}{2}\|\nabla u\|_{2}^{2}+\frac{3}{2} \int_{\mathbb{R}^{3}} V_{\infty} u^{2} \mathrm{~d} x-3 \int_{\mathbb{R}^{3}} F(u) \mathrm{d} x \\
& +\frac{q^{2}}{4 a} \int_{\mathbb{R}^{3}} \int_{\mathbb{R}^{3}}\left[5 \frac{1-e^{-\frac{|x-y|}{a}}}{|x-y| / a}+e^{-\frac{|x-y|}{a}}\right] u^{2}(x) u^{2}(y) \mathrm{d} x \mathrm{~d} y .
\end{aligned}
$$

We have the following corollary.

Corollary 1.6 Assume that (F1)-(F3) and (F4') hold. Then problem (1.5) admits a ground state solution $\bar{u} \in H^{1}\left(\mathbb{R}^{3}\right)$ such that

$$
\mathcal{I}^{\infty}(\bar{u})=\inf _{\mathcal{M}^{\infty}} \mathcal{I}^{\infty}=\inf _{u \in H^{1}\left(\mathbb{R}^{3}\right) \backslash\{0\}} \max _{t>0} \mathcal{I}^{\infty}\left(t^{2} u_{t}\right)>0 .
$$

Remark 1.7 Our more general conditions $(\mathrm{F} 1)-(\mathrm{F} 4)$ or $\left(\mathrm{F} 4^{\prime}\right)$ on the function $f(u)$ allow many other examples different to the pure power nonlinearity considered in [18]. For example, the function $f(u)=3|u| u \ln \left(1+u^{2}\right)+\frac{2|u|^{3} u}{1+u^{2}}$ satisfies (F1)-(F4). The function $f(u)=a|u|^{3 / 2} u+b|u|^{1 / 2} u$ with $a, b>0$ satisfies (F1)-(F3) and (F4') with $\theta=\frac{2}{3}$ when $15 \sqrt{10} a \geq 14 b^{3 / 2}>0$ but it does not fulfill (F4).

To prove Theorem 1.4, that is, to obtain a ground solution for Eq. (1.1) with (V1) and (V3), we first choose a minimizing sequence $\left\{u_{n}\right\}$ of $\mathcal{I}$ on $\mathcal{M}$, which satisfies

$$
\mathcal{I}\left(u_{n}\right) \rightarrow m:=\inf _{\mathcal{M}} \mathcal{I}, \quad \mathcal{P}\left(u_{n}\right)=0
$$

Next, we show that the sequence $\left\{u_{n}\right\}$ is bounded in $H^{1}\left(\mathbb{R}^{3}\right)$.

Due to lack of global compactness and adequate information on $\mathcal{I}^{\prime}\left(u_{n}\right)$ and in order to avoid relying the radial compactness, we establish a crucial inequality related to $\mathcal{I}(u), \mathcal{I}\left(u_{t}\right)$ and $\mathcal{J}(u)$ (Lemma 3.4), which plays a crucial role in our arguments, see Lemmas 3.8, 3.9, 3.13, 3.14 and 4.5. With the help of this inequality, we then can recover the compactness for the minimizing sequence $\left\{u_{n}\right\}$ and show that $\left\{u_{n}\right\}$ converges weakly to some $\bar{u} \in H^{1}\left(\mathbb{R}^{3}\right) \backslash\{0\}$ and $\mathcal{I}(\bar{u})=\inf _{\mathcal{M}} \mathcal{I}$ by using Lions' concentration-compactness, the "least energy squeeze approach" and some subtle analysis. Finally, we take advantage of a quantitative deformation lemma and the intermediate value theorem to show that $\bar{u}$ is a critical point of $\mathcal{I}$, as the Lagrange multiplier theorem does not work, because $\mathcal{M}$ is not a $\mathcal{C}^{1}$-manifold, .

To prove Theorem 1.1, we use the monotonicity technique explored by Jeanjean [21] to parameterize the nonlinearity $f$. In such a way, we build a parametrization of the energy functional associated to (1.1) and give some energy relations of problems (1.1) and (1.5) which play a key role in getting the critical point of (1.1), see Lemma 4.5. 
Moreover, in order to show that a critical point associated to the parametrization functional is indeed a solution to the original problem, we also need give a delicate estimation for the parametrization problem. Finally, we study the constant potential case by using weaker conditions.

Throughout the paper we make use of the following notations:

- Under (V1), $H^{1}\left(\mathbb{R}^{3}\right)$ denotes the Sobolev space equipped with the inner product and norm

$$
(u, v)=\int_{\mathbb{R}^{3}}[\nabla u \nabla v+V(x) u v] \mathrm{d} x, \quad\|u\|=(u, u)^{1 / 2}, \quad \forall u, v \in H^{1}\left(\mathbb{R}^{3}\right)
$$

- $L^{s}\left(\mathbb{R}^{3}\right)(1 \leq s<\infty)$ denotes the Lebesgue space with the norm $\|u\|_{s}=$ $\left(\int_{\mathbb{R}^{3}}|u|^{s} \mathrm{~d} x\right)^{\overline{1 / s}}$

- For any $x \in \mathbb{R}^{3}$ and $r>0, B_{r}(x):=\left\{y \in \mathbb{R}^{3}:|y-x|<r\right\}$;

- $S=\inf _{u \in D^{1,2}\left(\mathbb{R}^{3}\right) \backslash\{0\}}\|\nabla u\|_{2}^{2} /\|u\|_{6}^{2}$;

- $C_{1}, C_{2}, \cdots$ denote positive constants possibly different in different places.

\section{Variational setting}

We start with some preliminary basic results. Let us consider the nonlinear Schrödinger Lagrangian density

$$
\mathcal{L}_{\mathrm{Sc}}=i \hbar \bar{\psi} \partial_{t} \psi-\frac{\hbar^{2}}{2 m}|\nabla \psi|^{2}+2 F(\psi)
$$

where $\psi: \mathbb{R} \times \mathbb{R}^{3} \rightarrow \mathbb{C}, \hbar, m>0$, and let $(\phi, \mathbf{A})$ be the gauge potential of the electromagnetic field $(\mathbf{E}, \mathbf{H})$, namely $\phi: \mathbb{R}^{3} \rightarrow \mathbb{R}$ and $\mathbf{A}: \mathbb{R}^{3} \rightarrow \mathbb{R}^{3}$ satisfy

$$
\mathbf{E}=-\nabla \phi-\frac{1}{c} \partial_{t} \mathbf{A}, \quad \mathbf{H}=\nabla \times \mathbf{A}
$$

The coupling of the field $\psi$ with the electromagnetic field $(\mathbf{E}, \mathbf{H})$ through the minimal coupling rule, namely the study of the interaction between $\psi$ and its own electromagnetic field, can be obtained by replacing in $\mathcal{L}_{\mathrm{Sc}}$ the derivatives $\partial_{t}$ and $\nabla$ respectively with the covariant ones

$$
D_{t}=\partial_{t}+\frac{i q}{\hbar} \phi, \quad \mathbf{D}=\nabla-\frac{i q}{\hbar c} \mathbf{A}
$$

$q$ being a coupling constant. This leads to consider

$$
\begin{aligned}
\mathcal{L}_{\mathrm{CSc}} & =i \hbar \bar{\psi} D_{t} \psi-\frac{\hbar^{2}}{2 m}|\mathbf{D} \psi|^{2}+2 F(\psi) \\
& =i \hbar \bar{\psi}\left(\partial_{t}+\frac{i q}{\hbar} \phi\right) \psi-\frac{\hbar^{2}}{2 m}\left|\left(\nabla-\frac{i q}{\hbar c} \mathbf{A}\right) \psi\right|^{2}+2 F(\psi) .
\end{aligned}
$$


Now, to get the total Lagrangian density, we have to add to $\mathcal{L}_{\mathrm{CSc}}$ the Lagrangian density of the electromagnetic field.

The Bopp-Podolsky Lagrangian density (see [24, Formula (3.9)]) is

$$
\begin{aligned}
\mathcal{L}_{\mathrm{BP}}= & \frac{1}{8 \pi}\left\{|\mathbf{E}|^{2}-|\mathbf{H}|^{2}+a^{2}\left[(\operatorname{div} \mathbf{E})^{2}-\left|\nabla \times \mathbf{H}-\frac{1}{c} \partial_{t} \mathbf{E}\right|^{2}\right]\right\} \\
= & \frac{1}{8 \pi}\left\{\left|\nabla \phi+\frac{1}{c} \partial_{t} \mathbf{A}\right|^{2}-|\nabla \times \mathbf{A}|^{2}\right. \\
& \left.+a^{2}\left[\left(\Delta \phi+\frac{1}{c} \operatorname{div} \partial_{t} \mathbf{A}\right)^{2}-\left|\nabla \times \nabla \times \mathbf{A}+\frac{1}{c} \partial_{t}\left(\nabla \phi+\frac{1}{c} \partial_{t} \mathbf{A}\right)\right|^{2}\right]\right\} .
\end{aligned}
$$

Thus, the total action is

$$
\mathcal{S}(\psi, \phi, \mathbf{A})=\int_{\mathbb{R}^{3}} \mathcal{L} \mathrm{d} x \mathrm{~d} t
$$

where $\mathcal{L}:=\mathcal{L}_{\mathrm{CSc}}+\mathcal{L}_{\mathrm{BP}}$ is the total Lagrangian density.

Let $\mathcal{D}$ be the completion of $\mathcal{C}_{c}^{\infty}\left(\mathbb{R}^{3}\right)$ with respect to the norm $\|\cdot\|_{\mathcal{D}}$ induced by the scalar product

$$
\langle\varphi, \psi\rangle_{\mathcal{D}}:=\int_{\mathbb{R}^{3}} \nabla \varphi \nabla \psi \mathrm{d} x+a^{2} \int_{\mathbb{R}^{3}} \Delta \varphi \Delta \psi \mathrm{d} x
$$

Then $\mathcal{D}$ is a Hilbert space continuously embedded into $D^{1,2}\left(\mathbb{R}^{3}\right)$ and consequently in $L^{6}\left(\mathbb{R}^{3}\right)$.

We notice the following auxiliary properties; see Lemmas 3.1 and 3.2 in [18].

Lemma 2.1 The space $\mathcal{D}$ is continuously embedded in $L^{\infty}\left(\mathbb{R}^{3}\right)$.

The next property gives a useful characterization of the space $\mathcal{D}$.

Lemma 2.2 The space $\mathcal{C}_{c}^{\infty}\left(\mathbb{R}^{3}\right)$ is dense in

$$
\mathcal{A}:=\left\{\phi \in D^{1,2}\left(\mathbb{R}^{3}\right): \Delta \phi \in L^{2}\left(\mathbb{R}^{3}\right)\right\}
$$

normed by $\sqrt{\langle\phi, \phi\rangle_{\mathcal{D}}}$ and, therefore, $\mathcal{D}=\mathcal{A}$.

For every fixed $u \in H^{1}\left(\mathbb{R}^{3}\right)$, the Riesz representation theorem implies that there is a unique solution $\phi_{u} \in \mathcal{D}$ of the second equation in (1.1). To write explicitly such a solution (see also [24, Formula (2.6)]), we consider

$$
\mathcal{K}(x)=\frac{1-e^{-|x| / a}}{|x|} .
$$

We have the following fundamental properties. 
Lemma $2.3\left[18\right.$, Lemma 3.3] For all $y \in \mathbb{R}^{3}, \mathcal{K}(\cdot-y)$ solves in the sense of distributions

$$
-\Delta \phi+a^{2} \Delta^{2} \phi=4 \pi \delta_{y}
$$

\section{Moreover,}

(i) if $g \in L_{\text {loc }}^{1}\left(\mathbb{R}^{3}\right)$ and, for a.e. $x \in \mathbb{R}^{3}$, the map $y \in \mathbb{R}^{3} \mapsto g(y) /|x-y|$ is summable, then $\mathcal{K} * g \in L_{\text {loc }}^{1}\left(\mathbb{R}^{3}\right)$;

(ii) if $f \in L^{s}\left(\mathbb{R}^{3}\right)$ with $1 \leq s<3 / 2$, then $\mathcal{K} * g \in L^{q}\left(\mathbb{R}^{3}\right)$ for $q \in(3 s /(3-2 s),+\infty]$.

In both cases, $\mathcal{K} * g$ solves

$$
-\Delta \phi+a^{2} \Delta^{2} \phi=4 \pi g
$$

in the sense of distributions, and we have the following distributional derivatives:

$$
\nabla(\mathcal{K} * g)=(\nabla \mathcal{K}) * g \quad \text { and } \quad \Delta(\mathcal{K} * g)=(\Delta \mathcal{K}) * g \quad \text { a.e. in } \mathbb{R}^{3} .
$$

Fix $u \in H^{1}\left(\mathbb{R}^{3}\right)$, the unique solution in $\mathcal{D}$ of the second equation in (1.1) is

$$
\phi_{u}:=\mathcal{K} * u^{2}
$$

Actually the following useful properties hold.

Lemma 2.4 [18, Lemma 3.4] For every $u \in H^{1}\left(\mathbb{R}^{3}\right)$ we have:

(1) for every $y \in \mathbb{R}^{3}, \phi_{u(\cdot+y)}=\phi_{u}(\cdot+y)$;

(2) $\phi_{u} \geq 0$;

(3) for every $s \in(3,+\infty]$, $\phi_{u} \in L^{s}\left(\mathbb{R}^{3}\right) \cap \mathcal{C}_{0}\left(\mathbb{R}^{3}\right)$;

(4) for every $s \in(3 / 2,+\infty], \nabla \phi_{u}=\nabla \mathcal{K} * u^{2} \in L^{s}\left(\mathbb{R}^{3}\right) \cap \mathcal{C}_{0}\left(\mathbb{R}^{3}\right)$;

(5) $\phi_{u} \in \mathcal{D}$;

(6) $\left\|\phi_{u}\right\|_{6} \leq C\|u\|^{2}$;

(7) $\phi_{u}$ is the unique minimizer of the functional

$$
E(\phi)=\frac{1}{2}\|\nabla \phi\|_{2}^{2}+\frac{a^{2}}{2}\|\Delta \phi\|_{2}^{2}-\int_{\mathbb{R}^{3}} \phi u^{2} \mathrm{~d} x, \quad \phi \in \mathcal{D} .
$$

Moreover, if $v_{n} \rightarrow v$ in $H^{1}\left(\mathbb{R}^{3}\right)$, then $\phi_{v_{n}} \rightarrow \phi_{v}$ in $\mathcal{D}$.

Under hypotheses (V1), (F1) and (F2), the energy functional defined in $H^{1}\left(\mathbb{R}^{3}\right) \times \mathcal{D}$ by

$$
\begin{aligned}
\mathcal{S}(u, \phi)= & \frac{1}{2} \int_{\mathbb{R}^{3}}\left[|\nabla u|_{2}^{2}+V(x) u^{2}\right] \mathrm{d} x+\frac{q^{2}}{2} \int_{\mathbb{R}^{3}} \phi u^{2} \mathrm{~d} x \\
& -\frac{q^{2}}{16 \pi}\|\nabla \phi\|_{2}^{2}-\frac{a^{2} q^{2}}{16 \pi}\|\Delta \phi\|_{2}^{2}-\int_{\mathbb{R}^{3}} F(u) \mathrm{d} x
\end{aligned}
$$


is continuously differentiable and its critical points correspond to the weak solutions of problem (1.1). Indeed, if $(u, \phi) \in H^{1}\left(\mathbb{R}^{3}\right) \times \mathcal{D}$ is a critical point of $\mathcal{S}$, then

$$
\begin{aligned}
0= & \partial_{u} \mathcal{S}(u, \phi)[v]=\int_{\mathbb{R}^{3}}[\nabla u \nabla v+V(x) u v] \mathrm{d} x \\
& +q^{2} \int_{\mathbb{R}^{3}} \phi u v \mathrm{~d} x-\int_{\mathbb{R}^{3}} f(u) v \mathrm{~d} x, \quad \forall v \in H^{1}\left(\mathbb{R}^{3}\right)
\end{aligned}
$$

and

$$
0=\partial_{\phi} \mathcal{S}(u, \phi)[\xi]=\frac{q^{2}}{2} \int_{\mathbb{R}^{3}} u^{2} \xi \mathrm{d} x-\frac{q^{2}}{8 \pi} \int_{\mathbb{R}^{3}} \nabla \phi \nabla \xi \mathrm{d} x-\frac{a^{2} q^{2}}{8 \pi} \int_{\mathbb{R}^{3}} \Delta \phi \Delta \xi \mathrm{d} x, \quad \forall \xi \in \mathcal{D} .
$$

In order to avoid the difficulty generated by the strongly indefiniteness of the functional $\mathcal{S}$, we apply a reduction procedure. Noting that $\partial_{\phi} \mathcal{S}$ is a $\mathcal{C}^{1}$ functional, if $G_{\Phi}$ is the graph of the map $\Phi: u \in H^{1}\left(\mathbb{R}^{3}\right) \mapsto \phi_{u} \in \mathcal{D}$, an application of the implicit function theorem gives

$$
G_{\Phi}=\left\{(u, \phi) \in H^{1}\left(\mathbb{R}^{3}\right) \times \mathcal{D}: \partial_{\phi} \mathcal{S}(u, \phi)=0\right\} \quad \text { and } \quad \Phi \in \mathcal{C}^{1}\left(H^{1}\left(\mathbb{R}^{3}\right), \mathcal{D}\right) .
$$

Jointly with (2.3) and (2.4), the functional $\mathcal{I}(u):=\mathcal{S}\left(u, \phi_{u}\right)$ has the reduced form

$$
\mathcal{I}(u)=\frac{1}{2} \int_{\mathbb{R}^{3}}\left[|\nabla u|^{2}+V(x) u^{2}\right] \mathrm{d} x+\frac{q^{2}}{4} \int_{\mathbb{R}^{3}} \phi_{u} u^{2} \mathrm{~d} x-\int_{\mathbb{R}^{3}} F(u) \mathrm{d} x,
$$

which is of class $\mathcal{C}^{1}$ on $H^{1}\left(\mathbb{R}^{3}\right)$ and, for all $u, v \in H^{1}\left(\mathbb{R}^{3}\right)$

$$
\begin{aligned}
\mathcal{I}^{\prime}(u)[v] & =\partial_{u} \mathcal{S}(u, \Phi(u))[v]+\partial_{\phi} \mathcal{S}(u, \Phi(u)) \circ \Phi^{\prime}(u)[v] \\
& =\partial_{u} \mathcal{S}(u, \Phi(u))[v] \\
& =\int_{\mathbb{R}^{3}}[\nabla u \nabla v+V(x) u v] \mathrm{d} x+q^{2} \int_{\mathbb{R}^{3}} \phi_{u} u v \mathrm{~d} x-\int_{\mathbb{R}^{3}} f(u) v \mathrm{~d} x
\end{aligned}
$$

Moreover, the following statements are equivalent:

(i) the pair $(u, \phi) \in H^{1}\left(\mathbb{R}^{3}\right) \times \mathcal{D}$ is a critical point of $\mathcal{S}$, that is, $(u, \phi)$ is a solution of problem (1.1);

(ii) $u$ is a critical point of $\mathcal{I}$ and $\phi=\phi_{u}$.

Hence, if $u \in H^{1}\left(\mathbb{R}^{3}\right)$ is a critical point of $\mathcal{I}$, then the pair $\left(u, \phi_{u}\right)$ is a solution of (1.1). For the sake of simplicity, in many cases we just say $u \in H^{1}\left(\mathbb{R}^{3}\right)$, instead of $\left(u, \phi_{u}\right) \in H^{1}\left(\mathbb{R}^{3}\right) \times \mathcal{D}$, is a solution of $(1.1)$.

\section{Proof of Theorem 1.3}

In this section, we give the proof of Theorem 1.3. 
By a simple calculation, we have the following two lemmas.

Lemma 3.1 Let $b>0$. Then

$$
h(t):=t^{3}\left[e^{-\frac{b}{t}}-e^{-b}\right]+\frac{1-t^{3}}{3} b e^{-b} \geq 0, \quad \forall t>0
$$

and

$$
1-e^{-b}-\frac{1}{3} b e^{-b} \geq 0
$$

Lemma 3.2 (i) Assume that (V1) and (V3) hold. Then

$$
3\left[V(x)-t V\left(t^{-1} x\right)\right]-\left(1-t^{3}\right)[V(x)-\nabla V(x) \cdot x]>0, \quad \forall t \in[0,1) \cup(1,+\infty) .
$$

(ii) Assume that (F1) and (F4) hold. Then

$$
\frac{2\left(1-t^{3}\right)}{3} f(\tau) \tau+\left(t^{3}-2\right) F(\tau)+\frac{1}{t^{3}} F\left(t^{2} \tau\right) \geq 0, \quad \forall t>0, \tau \in \mathbb{R}
$$

(iii) Assume that (F1) and (F4') hold. Then

$$
\begin{aligned}
& \frac{2\left(1-t^{3}\right)}{3} f(\tau) \tau+\left(t^{3}-2\right) F(\tau)+\frac{1}{t^{3}} F\left(t^{2} \tau\right) \\
& +\frac{\theta_{0}}{6}(1-t)^{2}(2+t) V_{\infty} \tau^{2} \geq 0, \quad \forall t>0, \quad \tau \in \mathbb{R}
\end{aligned}
$$

Note that if $t \rightarrow 0$ in (3.4) and (3.5), then

$$
f(\tau) \tau-3 F(\tau) \geq 0, \quad \forall \tau \in \mathbb{R}
$$

and

$$
f(\tau) \tau-3 F(\tau)+\frac{\theta V_{\infty}}{2} \tau^{2} \geq 0, \quad \forall \tau \in \mathbb{R}
$$

Lemma 3.3 Assume that (V1) and (V3) hold. Then

$$
|\nabla V(x) \cdot x| \rightarrow 0 \text { as }|x| \rightarrow \infty
$$

Proof Arguing by contradiction, we assume that there exist a sequence $\left\{x_{n}\right\} \subset \mathbb{R}^{3}$ and $\delta>0$ such that

$$
\left|x_{n}\right| \rightarrow \infty \text {, and } \nabla V\left(x_{n}\right) \cdot x_{n} \geq \delta \text { or } \nabla V\left(x_{n}\right) \cdot x_{n} \leq-\delta \quad \forall n \in \mathbb{N} \text {. }
$$


Now, we distinguish two cases: i) $\nabla V\left(x_{n}\right) \cdot x_{n} \geq \delta$ for all $n \in \mathbb{N}$ and ii) $\nabla V\left(x_{n}\right) \cdot x_{n} \leq$ $-\delta$ for all $n \in \mathbb{N}$.

Case i) $\nabla V\left(x_{n}\right) \cdot x_{n} \geq \delta$ for all $n \in \mathbb{N}$. In this case, by (3.3), one has

$$
\begin{aligned}
\delta & \leq \nabla V\left(x_{n}\right) \cdot x_{n} \\
& <V\left(x_{n}\right)+\frac{3}{t^{3}-1}\left[V\left(x_{n}\right)-t V\left(t^{-1} x_{n}\right)\right] \\
& =V\left(x_{n}\right)+\frac{3(1-t)}{t^{3}-1} V\left(x_{n}\right)+\frac{3 t}{t^{3}-1}\left[V\left(x_{n}\right)-V\left(t^{-1} x_{n}\right)\right] \\
& =\frac{(t-1)(t+2)}{t^{2}+t+1} V\left(x_{n}\right)+\frac{3 t}{t^{3}-1}\left[V\left(x_{n}\right)-V\left(t^{-1} x_{n}\right)\right], \quad \forall t>1 .
\end{aligned}
$$

Since

$$
\lim _{|t| \rightarrow 1} \frac{(t-1)(t+2)}{t^{2}+t+1}=0
$$

there exists $t_{1}>1$ such that

$$
\frac{\left(t_{1}-1\right)\left(t_{1}+2\right)}{t_{1}^{2}+t_{1}+1} V_{\infty}<\frac{\delta}{2}
$$

Then it follows from (V1), (3.9) and (3.11) that

$$
\delta \leq \frac{\left(t_{1}-1\right)\left(t_{1}+2\right)}{t_{1}^{2}+t_{1}+1} V_{\infty}+\frac{3 t_{1}}{t_{1}^{3}-1}\left[V\left(x_{n}\right)-V\left(t_{1}^{-1} x_{n}\right)\right] \leq \frac{\delta}{2}+o(1)
$$

which is an obvious contradiction.

Case ii) $\nabla V\left(x_{n}\right) \cdot x_{n} \leq-\delta$ for all $n \in \mathbb{N}$. In this case, (3.3) gives

$$
\begin{aligned}
-\delta & \geq \nabla V\left(x_{n}\right) \cdot x_{n} \\
& >V\left(x_{n}\right)+\frac{3}{1-t^{3}}\left[t V\left(t^{-1} x_{n}\right)-V\left(x_{n}\right)\right] \\
& =V\left(x_{n}\right)+\frac{3(t-1)}{1-t^{3}} V\left(x_{n}\right)+\frac{3 t}{1-t^{3}}\left[V\left(t^{-1} x_{n}\right)-V\left(x_{n}\right)\right] \\
& =\frac{(t-1)(t+2)}{t^{2}+t+1} V\left(x_{n}\right)+\frac{3 t}{1-t^{3}}\left[V\left(t^{-1} x_{n}\right)-V\left(x_{n}\right)\right], \quad \forall 0<t<1 .
\end{aligned}
$$

From (3.10), there exists $0<t_{2}<1$ such that

$$
\frac{\left(t_{2}-1\right)\left(t_{2}+2\right)}{t_{2}^{2}+t_{2}+1} V_{\infty}>-\frac{\delta}{2}
$$


Then it follows from (V1), (3.13) and (3.14) that

$$
-\delta \geq \frac{\left(t_{2}-1\right)\left(t_{2}+2\right)}{t_{2}^{2}+t_{2}+1} V_{\infty}+\frac{3 t_{2}}{1-t_{2}^{3}}\left[V\left(t_{2}^{-1} x_{n}\right)-V\left(x_{n}\right)\right] \geq-\frac{\delta}{2}+o(1),
$$

which is again an obvious contradiction. This completes the proof.

Since $\mathcal{J}(u)=2 \mathcal{I}^{\prime}(u)[u]-\mathcal{P}(u)$ for $u \in H^{1}\left(\mathbb{R}^{3}\right)$, we have

$$
\begin{aligned}
\mathcal{J}(u)= & \frac{3}{2}\|\nabla u\|_{2}^{2}+\frac{1}{2} \int_{\mathbb{R}^{3}}[V(x)-\nabla V(x) \cdot x] u^{2} \mathrm{~d} x-\int_{\mathbb{R}^{3}}[2 f(u) u-3 F(u)] \mathrm{d} x \\
& +\frac{3 q^{2}}{4} \int_{\mathbb{R}^{3}} \phi_{u} u^{2} \mathrm{~d} x-\frac{q^{2}}{4 a} \int_{\mathbb{R}^{3}} \int_{\mathbb{R}^{3}} e^{-\frac{|x-y|}{a}} u^{2}(x) u^{2}(y) \mathrm{d} x \mathrm{~d} y .
\end{aligned}
$$

Define the function

$\beta(x, t):=3\left[V(x)-t V\left(t^{-1} x\right)\right]-\left(1-t^{3}\right)[V(x)-\nabla V(x) \cdot x], \quad \forall x \in \mathbb{R}^{3}, t>0$.

Lemma 3.4 Assume that (V1), (V3), (F1) and (F4) hold. Then

$$
\mathcal{I}(u) \geq \mathcal{I}\left(t^{2} u_{t}\right)+\frac{1-t^{3}}{3} \mathcal{J}(u)+\frac{1}{6} \int_{\mathbb{R}^{3}} \beta(x, t) u^{2} \mathrm{~d} x, \quad \forall u \in H^{1}\left(\mathbb{R}^{3}\right), t>0,
$$

where $u_{t}(x)=u(t x)$.

Proof For $u \in H^{1}\left(\mathbb{R}^{3}\right)$ and $t>0$, one has

$$
\begin{aligned}
\mathcal{I}\left(t^{2} u_{t}\right)= & \frac{t^{3}}{2}\|\nabla u\|_{2}^{2}+\frac{t}{2} \int_{\mathbb{R}^{3}} V\left(t^{-1} x\right) u^{2} \mathrm{~d} x \\
& +\frac{q^{2} t^{3}}{4} \int_{\mathbb{R}^{3}} \int_{\mathbb{R}^{3}} \frac{1-e^{-\frac{|x-y|}{t a}}}{|x-y|} u^{2}(x) u^{2}(y) \mathrm{d} x \mathrm{~d} y \\
& -\frac{1}{t^{3}} \int_{\mathbb{R}^{3}} F\left(t^{2} u\right) \mathrm{d} x
\end{aligned}
$$

Thus, (2.5), (3.1), (3.3), (3.4), (3.16), (3.17) and (3.19) imply that for all $u \in H^{1}\left(\mathbb{R}^{3}\right)$ and all $t>0$

$$
\begin{aligned}
& \mathcal{I}(u)-\mathcal{I}\left(t^{2} u_{t}\right) \\
& =\frac{1-t^{3}}{2}\|\nabla u\|_{2}^{2}+\frac{1}{2} \int_{\mathbb{R}^{3}}\left[V(x)-t V\left(t^{-1} x\right)\right] u^{2} \mathrm{~d} x+\int_{\mathbb{R}^{3}}\left[\frac{1}{t^{3}} F\left(t^{2} u\right)-F(u)\right] \mathrm{d} x
\end{aligned}
$$




$$
\begin{aligned}
& +\frac{q^{2}}{4} \int_{\mathbb{R}^{3}} \int_{\mathbb{R}^{3}} \frac{1-e^{-\frac{|x-y|}{a}}-t^{3}\left(1-e^{\frac{-|x-y|}{a t}}\right)}{|x-y|} u^{2}(x) u^{2}(y) \mathrm{d} x \mathrm{~d} y \\
& =\frac{1-t^{3}}{3} \mathcal{J}(u)+\frac{1}{6} \int_{\mathbb{R}^{3}}\left\{3\left[V(x)-t V\left(t^{-1} x\right)\right]-\left(1-t^{3}\right)[V(x)-\nabla V(x) \cdot x]\right\} u^{2} \mathrm{~d} x \\
& +\int_{\mathbb{R}^{3}}\left[\frac{2\left(1-t^{3}\right)}{3} f(u) u+\left(t^{3}-2\right) F(u)+\frac{1}{t^{3}} F\left(t^{2} u\right)\right] \mathrm{d} x \\
& +\frac{3 q^{2}}{4} \int_{\mathbb{R}^{3}} \int_{\mathbb{R}^{3}} \frac{t^{3}\left[e^{-\frac{|x-y|}{a t}}-e^{-\frac{|x-y|}{a}}\right]+\left(1-t^{3}\right) \frac{|x-y|}{3 a} e^{-\frac{|x-y|}{a}}}{|x-y|} u^{2}(x) u^{2}(y) \mathrm{d} x \mathrm{~d} y \\
& \geq \frac{1-t^{3}}{3} \mathcal{J}(u)+\frac{1}{6} \int_{\mathbb{R}^{3}} \beta(x, t) u^{2} \mathrm{~d} x \text {. }
\end{aligned}
$$

This shows (3.18).

Remark that (3.18) with $t \rightarrow 0$ gives

$$
\mathcal{I}(u) \geq \frac{1}{3} \mathcal{J}(u)+\frac{1}{6} \int_{\mathbb{R}^{3}}[2 V(x)+\nabla V(x) \cdot x] u^{2} \mathrm{~d} x, \quad \forall u \in H^{1}\left(\mathbb{R}^{3}\right) .
$$

For the limiting problem, corresponding to (2.5) and (3.16), we define the following functionals in $H^{1}\left(\mathbb{R}^{3}\right)$ :

$$
\mathcal{I}^{\infty}(u)=\frac{1}{2} \int_{\mathbb{R}^{3}}\left(|\nabla u|^{2}+V_{\infty} u^{2}\right) \mathrm{d} x+\frac{q^{2}}{4} \int_{\mathbb{R}^{3}} \phi_{u} u^{2} \mathrm{~d} x-\int_{\mathbb{R}^{3}} F(u) \mathrm{d} x
$$

and

$$
\begin{aligned}
\mathcal{J}^{\infty}(u)= & \frac{3}{2}\|\nabla u\|_{2}^{2}+\frac{V_{\infty}}{2}\|u\|_{2}^{2}-\int_{\mathbb{R}^{3}}[2 f(u) u-3 F(u)] \mathrm{d} x \\
& +\frac{3 q^{2}}{4} \int_{\mathbb{R}^{3}} \phi_{u} u^{2} \mathrm{~d} x-\frac{q^{2}}{4 a} \int_{\mathbb{R}^{3}} \int_{\mathbb{R}^{3}} e^{-\frac{|x-y|}{a} u^{2}(x) u^{2}(y) \mathrm{d} x \mathrm{~d} y}
\end{aligned}
$$

From Lemma 3.4, we deduce the following two properties.

Corollary 3.5 Assume that (V1), (V3), (F1) and (F4) hold. Then for $u \in \mathcal{M}$

$$
\mathcal{I}(u)=\max _{t>0} \mathcal{I}\left(t^{2} u_{t}\right)
$$

Corollary 3.6 Assume that (F1) and (F4) hold. Then

$$
\mathcal{I}^{\infty}(u) \geq \mathcal{I}^{\infty}\left(t^{2} u_{t}\right)+\frac{1-t^{3}}{3} \mathcal{J}^{\infty}(u)+\frac{(1-t)^{2}(2+t)}{6} V_{\infty}\|u\|_{2}^{2}, \quad \forall u \in H^{1}\left(\mathbb{R}^{3}\right), t>0 .
$$


By using (3.5) instead of (3.4), as in the proof of Lemma 3.4, we have the following lemma.

Lemma 3.7 Assume that $(\mathrm{F} 1)$ and $\left(F 4^{\prime}\right)$ hold. Then

$$
\begin{aligned}
\mathcal{I}^{\infty}(u) \geq & \mathcal{I}^{\infty}\left(t^{2} u_{t}\right)+\frac{1-t^{3}}{3} \mathcal{J}^{\infty}(u) \\
& +\frac{(1-\theta)(1-t)^{2}(2+t)}{6} V_{\infty}\|u\|_{2}^{2}, \quad \forall u \in H^{1}\left(\mathbb{R}^{3}\right), t>0 .
\end{aligned}
$$

Lemma 3.8 Assume that (V1), (V3) and (F1)-(F4) hold. Then for any $u \in$ $H^{1}\left(\mathbb{R}^{3}\right) \backslash\{0\}$, there exists a unique $t_{u}>0$ such that $t_{u}^{2} u_{t_{u}} \in \mathcal{M}$.

Proof Let $u \in H^{1}\left(\mathbb{R}^{3}\right) \backslash\{0\}$ be fixed and define the function $\zeta(t):=\mathcal{I}\left(t^{2} u_{t}\right)$ on $(0, \infty)$. Using (3.16) and (1.6), it is easily checked that

$$
\zeta^{\prime}(t)=0 \Leftrightarrow \frac{1}{t} \mathcal{J}\left(t^{2} u_{t}\right)=0 \Leftrightarrow t^{2} u_{t} \in \mathcal{M} .
$$

By (V1) and (F1)-(F3), we have $\lim _{t \rightarrow 0^{+}} \zeta(t)=0, \zeta(t)>0$ for $t>0$ small and $\zeta(t)<0$ for $t$ large. Therefore, $\max _{t \in(0, \infty)} \zeta(t)$ is achieved at $t_{0}=t_{u}>0$, so that $\zeta^{\prime}\left(t_{0}\right)=0$ and $t_{0}^{2} u_{t_{0}} \in \mathcal{M}$.

Next, we claim that $t_{u}$ is unique for any $u \in H^{1}\left(\mathbb{R}^{3}\right) \backslash\{0\}$. In fact, for any given $u \in H^{1}\left(\mathbb{R}^{3}\right) \backslash\{0\}$, let $t_{1}, t_{2}>0$ be such that $\zeta^{\prime}\left(t_{1}\right)=\zeta^{\prime}\left(t_{2}\right)=0$. Then $\mathcal{J}\left(t_{1}^{2} u_{t_{1}}\right)=$ $\mathcal{J}\left(t_{2}^{2} u_{t_{2}}\right)=0$. Jointly with (3.18), we have

$$
\begin{aligned}
\mathcal{I}\left(t_{1}^{2} u_{t_{1}}\right) & \geq \mathcal{I}\left(t_{2}^{2} u_{t_{2}}\right)+\frac{t_{1}^{3}-t_{2}^{3}}{3 t_{1}^{3}} \mathcal{J}\left(t_{1}^{2} u_{t_{1}}\right)+\frac{t_{1}}{6} \int_{\mathbb{R}^{3}} \beta\left(x, t_{2} / t_{1}\right) u^{2} \mathrm{~d} x \\
& =\mathcal{I}\left(t_{2}^{2} u_{t_{2}}\right)+\frac{t_{1}}{6} \int_{\mathbb{R}^{3}} \beta\left(x, t_{2} / t_{1}\right) u^{2} \mathrm{~d} x
\end{aligned}
$$

and

$$
\begin{aligned}
\mathcal{I}\left(t_{2}^{2} u_{t_{2}}\right) & \geq \mathcal{I}\left(t_{1}^{2} u_{t_{1}}\right)+\frac{t_{2}^{3}-t_{1}^{3}}{3 t_{2}^{3}} \mathcal{J}\left(t_{2}^{2} u_{t_{2}}\right)+\frac{t_{2}}{6} \int_{\mathbb{R}^{3}} \beta\left(x, t_{1} / t_{2}\right) u^{2} \mathrm{~d} x \\
& =\mathcal{I}\left(t_{1}^{2} u_{t_{1}}\right)+\frac{t_{2}}{6} \int_{\mathbb{R}^{3}} \beta\left(x, t_{1} / t_{2}\right) u^{2} \mathrm{~d} x .
\end{aligned}
$$

Then (3.1), (3.25) and (3.25) give $t_{1}=t_{2}$. Therefore, $t_{u}>0$ is unique for any $u \in H^{1}\left(\mathbb{R}^{3}\right) \backslash\{0\}$.

Combining Corollary 3.5 with Lemma 3.8, w obtain the following min-max property.

Lemma 3.9 Assume that (V1), (V3) and (F1)-(F4) hold. Then

$$
m=\inf _{\mathcal{M}} \mathcal{I}=\inf _{u \in H^{1}\left(\mathbb{R}^{3}\right) \backslash\{0\}} \max _{t>0} \mathcal{I}\left(t^{2} u_{t}\right) .
$$


Lemma 3.10 Assume that (V1), (V3) and (F1)-(F4) hold. Then

(i) there exists $\rho>0$ such that $\|u\| \geq \rho, \forall u \in \mathcal{M}$;

(ii) $m=\inf _{\mathcal{M}} \mathcal{I}>0$.

Proof (i). In view of [13, Lemma 2.5], if $V$ satisfies (V1) and (V3), then there exist $\varrho_{1}, \varrho_{2}>0$ such that

$$
\begin{aligned}
& 2 V(x)+\nabla V(x) \cdot x \geq \varrho_{1}, \quad \forall x \in \mathbb{R}^{3}, \\
& V(x)-\nabla V(x) \cdot x \geq \varrho_{2}, \quad \forall x \in \mathbb{R}^{3} .
\end{aligned}
$$

Since $\mathcal{J}(u)=0$ for $u \in \mathcal{M}$, by (3.2), (3.16), (3.28) and the Sobolev embedding theorem, we have

$$
\begin{aligned}
\frac{\min \left\{3, \varrho_{2}\right\}}{2}\|u\|^{2} \leq & \frac{3}{2}\|\nabla u\|_{2}^{2}+\frac{1}{2} \int_{\mathbb{R}^{3}}[V(x)-\nabla V(x) \cdot x] u^{2} \mathrm{~d} x \\
& +\frac{3 q^{2}}{4} \int_{\mathbb{R}^{3}} \int_{\mathbb{R}^{3}} \frac{1-e^{-\frac{|x-y|}{a}}-\frac{|x-y|}{3 a} e^{-\frac{|x-y|}{a}}}{|x-y|} u^{2}(x) u^{2}(y) \mathrm{d} x \mathrm{~d} y \\
\leq & \int_{\mathbb{R}^{3}}[2 f(u) u-3 F(u)] \mathrm{d} x \\
\leq & \frac{\min \left\{3, \varrho_{2}\right\}}{4}\|u\|^{2}+C_{1}\|u\|^{p}, \quad \forall u \in \mathcal{M},
\end{aligned}
$$

which implies

$$
\|u\| \geq \rho:=\left(\frac{\min \left\{3, \varrho_{2}\right\}}{4 C_{1}}\right)^{1 /(p-2)}, \quad \forall u \in \mathcal{M} .
$$

(ii). Let $\left\{u_{n}\right\} \subset \mathcal{M}$ be such that $\mathcal{I}\left(u_{n}\right) \rightarrow m$. There are two possible cases: 1$)$ $\inf _{n \in \mathbb{N}}\left\|u_{n}\right\|_{2}>0$ and 2) $\inf _{n \in \mathbb{N}}\left\|u_{n}\right\|_{2}=0$.

Case 1) $\inf _{n \in \mathbb{N}}\left\|u_{n}\right\|_{2}:=\rho_{1}>0$. In this case, (3.20) and (3.27) yield

$$
m+o(1)=\mathcal{I}\left(u_{n}\right)=\mathcal{I}\left(u_{n}\right)-\frac{1}{3} \mathcal{J}\left(u_{n}\right) \geq \frac{\varrho_{1}}{6} \rho_{1}^{2}>0 .
$$

Case 2) $\inf _{n \in \mathbb{N}}\left\|u_{n}\right\|_{2}=0$. By (3.29), passing to a subsequence, we have

$$
\left\|u_{n}\right\|_{2} \rightarrow 0, \quad\left\|\nabla u_{n}\right\|_{2} \geq \frac{1}{2} \rho .
$$

Let $t_{n}=\left\|\nabla u_{n}\right\|_{2}^{-2 / 3}$. Then (3.31) implies that $\left\{t_{n}\right\}$ is bounded. Using (F1), (F2) and the Sobolev inequality, there exists $C_{2}>0$ such that

$$
\left|\int_{\mathbb{R}^{3}} F(u) \mathrm{d} x\right| \leq C_{2}\|u\|_{2}^{2}+\frac{S^{3}}{4}\|u\|_{6}^{6} \leq C_{2}\|u\|_{2}^{2}+\frac{1}{4}\|\nabla u\|_{2}^{6}, \quad \forall u \in H^{1}\left(\mathbb{R}^{3}\right) .
$$

Since $\mathcal{J}\left(u_{n}\right)=0$ for all $n \in \mathbb{N}$, then (3.18), (3.19), (3.31) and (3.32) give 


$$
\begin{aligned}
m+o(1)= & \mathcal{I}\left(u_{n}\right) \geq \mathcal{I}\left(t_{n}^{2}\left(u_{n}\right)_{t_{n}}\right) \\
= & \frac{t_{n}^{3}}{2}\left\|\nabla u_{n}\right\|_{2}^{2}+\frac{t_{n}}{2} \int_{\mathbb{R}^{3}} V\left(t_{n}^{-1} x\right) u_{n}^{2} \mathrm{~d} x \\
& +\frac{q^{2} t_{n}^{3}}{4} \int_{\mathbb{R}^{3}} \int_{\mathbb{R}^{3}} \frac{1-e^{-\frac{|x-y|}{a t_{n}}}}{|x-y|} u_{n}^{2}(x) u_{n}^{2}(y) \mathrm{d} x \mathrm{~d} y \\
& \quad-\frac{1}{t_{n}^{3}} \int_{\mathbb{R}^{3}} F\left(t_{n}^{2} u_{n}\right) \mathrm{d} x \\
\geq & \frac{t_{n}^{3}}{2}\left\|\nabla u_{n}\right\|_{2}^{2}-C_{2} t_{n}\left\|u_{n}\right\|_{2}^{2}-\frac{t_{n}^{9}}{4}\left\|\nabla u_{n}\right\|_{2}^{6} \\
= & \frac{1}{4} t_{n}^{3}\left\|\nabla u_{n}\right\|_{2}^{2}\left[2-\left(t_{n}^{3}\left\|\nabla u_{n}\right\|_{2}^{2}\right)^{2}\right]+o(1)=\frac{1}{4}+o(1) .
\end{aligned}
$$

Cases 1) and 2) show that $m=\inf _{\mathcal{M}} \mathcal{I}>0$. This completes the proof.

Lemma 3.11 Assume that (V1), (V3) and (F1)-(F4) hold. Then $m^{\infty}:=\inf _{\mathcal{M}^{\infty}} \mathcal{I}^{\infty} \geq$ $m$.

Proof Arguing by contradiction, suppose that $m>m^{\infty}$. Let $\varepsilon:=m-m^{\infty}$. Then there exists $u_{\varepsilon}^{\infty}$ such that

$$
u_{\varepsilon}^{\infty} \in \mathcal{M}^{\infty} \text { and } m^{\infty}+\frac{\varepsilon}{2}>\mathcal{I}^{\infty}\left(u_{\varepsilon}^{\infty}\right)
$$

In view of Lemma 3.8, there exists $t_{\varepsilon}>0$ such that $t_{\varepsilon}^{2}\left(u_{\varepsilon}^{\infty}\right)_{t_{\varepsilon}} \in \mathcal{M}$. Thus, it follows from (V1), (2.5), (3.3), (3.21), (3.24) and (3.33) that

$$
m^{\infty}+\frac{\varepsilon}{2}>\mathcal{I}^{\infty}\left(u_{\varepsilon}^{\infty}\right) \geq \mathcal{I}^{\infty}\left(t_{\varepsilon}^{2}\left(u_{\varepsilon}^{\infty}\right)_{t_{\varepsilon}}\right) \geq \mathcal{I}\left(t_{\varepsilon}^{2}\left(u_{\varepsilon}^{\infty}\right)_{t_{\varepsilon}}\right) \geq m
$$

This contradiction shows that $m^{\infty} \geq m$.

By combining [18, Lemma B.2] and [23,26], we obtain the following Brezis-Lieb type lemma, see [8].

Lemma 3.12 Assume that (V1), (V2), (F1) and (F2) hold. If $u_{n} \rightarrow \bar{u}$ in $H^{1}\left(\mathbb{R}^{3}\right)$, then up to a subsequence

$$
\begin{aligned}
\mathcal{I}\left(u_{n}\right) & =\mathcal{I}(\bar{u})+\mathcal{I}\left(u_{n}-\bar{u}\right)+o(1), \\
\mathcal{J}\left(u_{n}\right) & =\mathcal{J}(\bar{u})+\mathcal{J}\left(u_{n}-\bar{u}\right)+o(1) \\
\mathcal{I}^{\prime}\left(u_{n}\right) & =\mathcal{I}^{\prime}(\bar{u})+\mathcal{I}^{\prime}\left(u_{n}-\bar{u}\right)+o(1), \\
\mathcal{I}^{\prime}\left(u_{n}\right)\left[u_{n}\right] & =\mathcal{I}^{\prime}(\bar{u})[\bar{u}]+\mathcal{I}^{\prime}\left(u_{n}-\bar{u}\right)\left[u_{n}-\bar{u}\right]+o(1) .
\end{aligned}
$$

Lemma 3.13 Assume that (V1), (V3) and (F1)-(F4) hold. Then $m$ is achieved. 
Proof Let $\left\{u_{n}\right\} \subset \mathcal{M}$ be such that $\mathcal{I}\left(u_{n}\right) \rightarrow m$. Since $\mathcal{J}\left(u_{n}\right)=0$, then (3.20) and (3.27) yield

$$
\begin{aligned}
m+o(1) & =\mathcal{I}\left(u_{n}\right)=\mathcal{I}\left(u_{n}\right)-\frac{1}{3} \mathcal{J}\left(u_{n}\right) \\
& \geq \frac{1}{6} \int_{\mathbb{R}^{3}}[2 V(x)+\nabla V(x) \cdot x] u_{n}^{2} \mathrm{~d} x \geq \frac{\varrho_{1}}{6}\left\|u_{n}\right\|_{2}^{2} .
\end{aligned}
$$

This shows that $\left\{\left\|u_{n}\right\|_{2}\right\}$ is bounded. Now we assert that $\left\{\left\|\nabla u_{n}\right\|_{2}\right\}$ is also bounded. Arguing by contradiction, suppose that $\left\|\nabla u_{n}\right\|_{2} \rightarrow \infty$. From (F1), (F2) and the Sobolev inequality, there exists $C_{2}>0$ such that

$$
\begin{aligned}
\left|\int_{\mathbb{R}^{3}} F(u) \mathrm{d} x\right| \leq & C_{2}\|u\|_{2}^{2}+\frac{1}{2(8 m)^{2}} S^{3}\|u\|_{6}^{6} \leq C_{2}\|u\|_{2}^{2} \\
& +\frac{1}{2(8 m)^{2}}\|\nabla u\|_{2}^{6}, \quad \forall u \in H^{1}\left(\mathbb{R}^{3}\right) .
\end{aligned}
$$

Let $t_{n}=\left(8 m /\left\|\nabla u_{n}\right\|_{2}^{2}\right)^{1 / 3}$. Since $\mathcal{J}\left(u_{n}\right)=0$, it follows from (3.18), (3.19) and (3.38) that

$$
\begin{aligned}
m+o(1)= & \mathcal{I}\left(u_{n}\right) \geq \mathcal{I}\left(t_{n}^{2}\left(u_{n}\right)_{t_{n}}\right) \\
= & \frac{t_{n}^{3}}{2}\left\|\nabla u_{n}\right\|_{2}^{2}+\frac{t_{n}}{2} \int_{\mathbb{R}^{3}} V\left(t_{n}^{-1} x\right) u_{n}^{2} \mathrm{~d} x \\
& +\frac{q^{2} t_{n}^{3}}{4} \int_{\mathbb{R}^{3}} \int_{\mathbb{R}^{3}} \frac{1-e^{-\frac{|x-y|}{a t_{n}}}}{|x-y|} u_{n}^{2}(x) u_{n}^{2}(y) \mathrm{d} x \mathrm{~d} y \\
& -\frac{1}{t_{n}^{3}} \int_{\mathbb{R}^{3}} F\left(t_{n}^{2} u_{n}\right) \mathrm{d} x \\
\geq & \frac{t_{n}^{3}}{2}\left\|\nabla u_{n}\right\|_{2}^{2}-C_{2} t_{n}\left\|u_{n}\right\|_{2}^{2}-\frac{1}{4(8 m)^{2}}\left(t_{n}^{3}\left\|\nabla u_{n}\right\|_{2}^{2}\right)^{3} \\
= & \frac{1}{2} t_{n}^{3}\left\|\nabla u_{n}\right\|_{2}^{2}\left[1-\frac{1}{2}\left(\frac{t_{n}^{3}\left\|\nabla u_{n}\right\|_{2}^{2}}{8 m}\right)^{2}\right]+o(1) \\
= & 2 m+o(1) .
\end{aligned}
$$

This contradiction shows that $\left\{\left\|\nabla u_{n}\right\|_{2}\right\}$ is also bounded and the assertion holds. Hence $\left\{u_{n}\right\}$ is bounded in $H^{1}\left(\mathbb{R}^{3}\right)$. Thus, there exists $\bar{u} \in H^{1}\left(\mathbb{R}^{3}\right)$ such that, passing to a subsequence, $u_{n} \rightarrow \bar{u}$ in $H^{1}\left(\mathbb{R}^{3}\right), u_{n} \rightarrow \bar{u}$ in $L_{\text {loc }}^{s}\left(\mathbb{R}^{3}\right)$ for all $1 \leq s<6$ and $u_{n} \rightarrow \bar{u}$ a.e. in $\mathbb{R}^{3}$. There are two possible cases: i) $\bar{u}=0$ and ii) $\bar{u} \neq 0$.

Case i) $\bar{u}=0$, i.e. $u_{n} \rightarrow 0$ in $H^{1}\left(\mathbb{R}^{3}\right), u_{n} \rightarrow 0$ in $L_{\text {loc }}^{s}\left(\mathbb{R}^{3}\right)$ for all $1 \leq s<6$ and $u_{n} \rightarrow 0$ a.e. in $\mathbb{R}^{3}$. Using (V1) and (3.8), it is easily checked that

$$
\lim _{n \rightarrow \infty} \int_{\mathbb{R}^{3}}\left[V_{\infty}-V(x)\right] u_{n}^{2} \mathrm{~d} x=\lim _{n \rightarrow \infty} \int_{\mathbb{R}^{3}} \nabla V(x) \cdot x u_{n}^{2} \mathrm{~d} x=0
$$


From (2.5), (3.16), (3.21), (3.22) and (3.40), we derive

$$
\mathcal{I}^{\infty}\left(u_{n}\right) \rightarrow m \text { and } \mathcal{J}^{\infty}\left(u_{n}\right) \rightarrow 0
$$

From [26, Lemma 1.21], we deduce that there exist $\delta>0$ and a sequence $\left\{y_{n}\right\} \subset \mathbb{R}^{3}$ such that $\int_{B_{1}\left(y_{n}\right)}\left|u_{n}\right|^{2} \mathrm{~d} x>\delta$. Let $\hat{u}_{n}(x)=u_{n}\left(x+y_{n}\right)$. Then we have $\left\|\hat{u}_{n}\right\|=\left\|u_{n}\right\|$ and

$$
\mathcal{J}^{\infty}\left(\hat{u}_{n}\right)=o(1), \quad \mathcal{I}^{\infty}\left(\hat{u}_{n}\right) \rightarrow m, \quad \int_{B_{1}(0)}\left|\hat{u}_{n}\right|^{2} \mathrm{~d} x>\delta
$$

Therefore, there exists $\hat{u} \in H^{1}\left(\mathbb{R}^{3}\right) \backslash\{0\}$ such that, passing to a subsequence,

$$
\left\{\begin{array}{l}
\hat{u}_{n} \rightarrow \hat{u}, \quad \text { in } H^{1}\left(\mathbb{R}^{3}\right) ; \\
\hat{u}_{n} \rightarrow \hat{u}, \text { in } L_{\text {loc }}^{s}\left(\mathbb{R}^{3}\right), \forall s \in[1,6) \\
\hat{u}_{n} \rightarrow \hat{u}, \text { a.e. in } \mathbb{R}^{3}
\end{array}\right.
$$

Let $w_{n}=\hat{u}_{n}-\hat{u}$. Then (3.43) and Lemma 3.12 yield

$$
\mathcal{I}^{\infty}\left(\hat{u}_{n}\right)=\mathcal{I}^{\infty}(\hat{u})+\mathcal{I}^{\infty}\left(w_{n}\right)+o(1), \quad \mathcal{J}^{\infty}\left(\hat{u}_{n}\right)=\mathcal{J}^{\infty}(\hat{u})+\mathcal{J}^{\infty}\left(w_{n}\right)+o(1)
$$

We define the functional $\Psi^{\infty}: H^{1}\left(\mathbb{R}^{3}\right) \rightarrow \mathbb{R}$ by

$$
\begin{aligned}
\Psi^{\infty}(u)= & \mathcal{I}^{\infty}(u)-\frac{1}{3} \mathcal{J}^{\infty}(u) \\
= & \frac{V_{\infty}}{3}\|u\|_{2}^{2}+\frac{2}{3} \int_{\mathbb{R}^{3}}[f(u) u-3 F(u)] \mathrm{d} x \\
& +\frac{q^{2}}{12 a} \int_{\mathbb{R}^{3}} \int_{\mathbb{R}^{3}} e^{-\frac{|x-y|}{a}} u^{2}(x) u^{2}(y) \mathrm{d} x \mathrm{~d} y .
\end{aligned}
$$

By (3.21), (3.22), (3.42), (3.44) and (3.45), we have

$$
\Psi^{\infty}\left(w_{n}\right)=m-\Psi^{\infty}(\hat{u})+o(1), \text { and } \mathcal{J}^{\infty}\left(w_{n}\right)=-\mathcal{J}^{\infty}(\hat{u})+o(1)
$$

If there exists a subsequence $\left\{w_{n_{i}}\right\}$ of $\left\{w_{n}\right\}$ such that $w_{n_{i}}=0$, then

$$
\mathcal{I}^{\infty}(\hat{u})=m \text { and } \mathcal{J}^{\infty}(\hat{u})=0
$$

Thus, we assume that $w_{n} \neq 0$ for all $n \in \mathbb{N}$. We claim that $\mathcal{J}^{\infty}(\hat{u}) \leq 0$. Otherwise, if $\mathcal{J}^{\infty}(\hat{u})>0$, then (3.46) implies $\mathcal{J}^{\infty}\left(w_{n}\right)<0$ for large $n$. In view of Lemma 3.8, there exists $t_{n}>0$ such that $t_{n}^{2}\left(w_{n}\right)_{t_{n}} \in \mathcal{M}^{\infty}$ for large $n$. From (3.21), (3.22), (3.23), (3.46) and Lemma 3.11, we obtain 


$$
\begin{aligned}
m-\Psi^{\infty}(\hat{u})+o(1) & =\Psi^{\infty}\left(w_{n}\right)=\mathcal{I}^{\infty}\left(w_{n}\right)-\frac{1}{3} \mathcal{J}^{\infty}\left(w_{n}\right) \\
& \geq \mathcal{I}^{\infty}\left(t_{n}^{2}\left(w_{n}\right)_{t_{n}}\right)-\frac{t_{n}^{3}}{3} \mathcal{J}^{\infty}\left(w_{n}\right)+\frac{\left(1-t_{n}\right)^{2}\left(2+t_{n}\right) V_{\infty}}{6}\left\|w_{n}\right\|_{2}^{2} \\
& \geq m^{\infty}-\frac{t_{n}^{3}}{3} \mathcal{J}^{\infty}\left(w_{n}\right)+\frac{\left(1-t_{n}\right)^{2}\left(2+t_{n}\right) V_{\infty}}{6}\left\|w_{n}\right\|_{2}^{2} \\
& >m
\end{aligned}
$$

which contradicts the fact that $\Psi^{\infty}(\hat{u})>0$. Hence, $\mathcal{J}^{\infty}(\hat{u}) \leq 0$ and the claim holds. In view of Lemma 3.8, there exists $t_{\infty}>0$ such that $t_{\infty}^{2} \hat{u}_{t_{\infty}} \in \mathcal{M}^{\infty}$. Now (3.23), (3.41), (3.42), (3.45), Fatou's lemma and Lemma 3.11 yield

$$
\begin{aligned}
m & =\lim _{n \rightarrow \infty}\left[\mathcal{I}^{\infty}\left(\hat{u}_{n}\right)-\frac{1}{3} \mathcal{J}^{\infty}\left(\hat{u}_{n}\right)\right] \\
& =\lim _{n \rightarrow \infty} \Psi^{\infty}\left(\hat{u}_{n}\right) \geq \Psi^{\infty}(\hat{u})=\mathcal{I}^{\infty}(\hat{u})-\frac{1}{3} \mathcal{J}^{\infty}(\hat{u}) \\
& \geq \mathcal{I}^{\infty}\left(t_{\infty}^{2} \hat{u}_{t_{\infty}}\right)-\frac{t_{\infty}^{3}}{3} \mathcal{J}^{\infty}(\hat{u})+\frac{\left(1-t_{\infty}\right)^{2}\left(2+t_{\infty}\right) V_{\infty}}{6}\|\hat{u}\|_{2}^{2} \\
& \geq m^{\infty}-\frac{t_{\infty}^{3}}{3} \mathcal{J}^{\infty}(\hat{u})+\frac{\left(1-t_{\infty}\right)^{2}\left(2+t_{\infty}\right) V_{\infty}}{6}\|\hat{u}\|_{2}^{2} \geq m,
\end{aligned}
$$

which implies again the validity of (3.47) also in this case. In view of Lemma 3.8, there exists $\hat{t}>0$ such that $\hat{t}^{2} \hat{u}_{\hat{t}} \in \mathcal{M}$. Moreover, it follows from (V1), (2.5), (3.21), (3.47) and Corollary 3.5 that

$$
m \leq \mathcal{I}\left(\hat{t}^{2} \hat{u}_{\hat{t}}\right) \leq \mathcal{I}^{\infty}\left(\hat{t}^{2} \hat{u}_{\hat{t}}\right) \leq \mathcal{I}^{\infty}(\hat{u})=m
$$

This shows that $m$ is achieved at $\hat{t}^{2} \hat{u}_{\hat{t}} \in \mathcal{M}$.

Case ii) $\bar{u} \neq 0$. We define the functional $\Psi: H^{1}\left(\mathbb{R}^{3}\right) \rightarrow \mathbb{R}$ by

$$
\begin{aligned}
\Psi(u)= & \mathcal{I}(u)-\frac{1}{3} \mathcal{J}(u) \\
= & \frac{1}{6} \int_{\mathbb{R}^{3}}[2 V(x)+\nabla V(x) \cdot x] u_{n}^{2} \mathrm{~d} x+\frac{2}{3} \int_{\mathbb{R}^{3}}[f(u) u-3 F(u)] \mathrm{d} x \\
& +\frac{q^{2}}{12 a} \int_{\mathbb{R}^{3}} \int_{\mathbb{R}^{3}} e^{-\frac{|x-y|}{a}} u^{2}(x) u^{2}(y) \mathrm{d} x \mathrm{~d} y .
\end{aligned}
$$

In this case, similarly to the proof of (3.47), by using $\mathcal{I}, \mathcal{J}$ and $\Psi$ instead of $\mathcal{I}^{\infty}, \mathcal{J}^{\infty}$ and $\Psi^{\infty}$, we deduce that $\mathcal{I}(\bar{u})=m$ and $\mathcal{J}(\bar{u})=0$.

Lemma 3.14 Assume that (V1), (V3) and (F1)-(F4) hold. If $\bar{u} \in \mathcal{M}$ and $\mathcal{I}(\bar{u})=m$, then $\bar{u}$ is a critical point of $\mathcal{I}$.

Proof Assume that $\mathcal{I}^{\prime}(\bar{u}) \neq 0$. Then there exist $\delta>0$ and $\rho>0$ such that

$$
\|u-\bar{u}\| \leq 3 \delta \Rightarrow\left\|\mathcal{I}^{\prime}(u)\right\| \geq \rho
$$


It is easy to check that

$$
\lim _{t \rightarrow 1}\left\|t^{2} \bar{u}_{t}-\bar{u}\right\|=0
$$

Then there exists $\delta_{1}>0$ such that

$$
|t-1|<\delta_{1} \Rightarrow\left\|t^{2} \bar{u}_{t}-\bar{u}\right\|<\delta .
$$

Using (V1), (V3) and (F1)-(F3), it is easy to prove that there exist $T_{1} \in(0,1)$ and $T_{2} \in(1, \infty)$ such that

$$
\mathcal{J}\left(T_{1}^{2} \bar{u}_{T_{1}}\right)>0, \quad \mathcal{J}\left(T_{2}^{2} \bar{u}_{T_{2}}\right)<0
$$

In view of Lemma 3.4, we have

$$
\mathcal{I}\left(t^{2} \bar{u}_{t}\right) \leq \mathcal{I}(\bar{u})-\frac{1}{6} \int_{\mathbb{R}^{3}} \beta(x, t) \bar{u}^{2} \mathrm{~d} x, \quad \forall t>0 .
$$

The rest of the proof is similar to that of [11, Lemma 2.14]. For the sake of completeness, we give the details. Let

$$
\beta_{0}:=\min \left\{\int_{\mathbb{R}^{3}} \beta\left(T_{1}, x\right) \bar{u}^{2} \mathrm{~d} x, \int_{\mathbb{R}^{3}} \beta\left(T_{2}, x\right) \bar{u}^{2} \mathrm{~d} x\right\},
$$

and $\varepsilon:=\min \left\{\beta_{0} / 24,1, \rho \delta / 8\right\}$. From [26, Lemma 2.3], there exists a deformation $\eta \in \mathcal{C}\left([0,1] \times H^{1}\left(\mathbb{R}^{3}\right), H^{1}\left(\mathbb{R}^{3}\right)\right)$ such that

(i) $\eta(1, u)=u$ if $\mathcal{I}(u)<m-2 \varepsilon$ or $\mathcal{I}(u)>m+2 \varepsilon$;

(ii) $\eta\left(1, \mathcal{I}^{m+\varepsilon} \cap B(\bar{u}, \delta)\right) \subset \mathcal{I}^{m-\varepsilon}$;

(iii) $\mathcal{I}(\eta(1, u)) \leq \mathcal{I}(u), \forall u \in H^{1}\left(\mathbb{R}^{3}\right)$;

(iv) $\eta(1, u)$ is a homeomorphism of $H^{1}\left(\mathbb{R}^{3}\right)$.

Note that Corollary 3.5 implies that $\mathcal{I}\left(t^{2} \bar{u}_{t}\right) \leq \mathcal{I}(\bar{u})=m$ for all $t>0$. Then (3.49) and ii) give

$$
\mathcal{I}\left(\eta\left(1, t^{2} \bar{u}_{t}\right)\right) \leq m-\varepsilon, \quad \forall t>0, \quad|t-1|<\delta_{1} .
$$

On the other hand, (3.51) and iii) yield

$$
\begin{aligned}
\mathcal{I}\left(\eta\left(1, t^{2} \bar{u}_{t}\right)\right) & \leq \mathcal{I}\left(t^{2} \bar{u}_{t}\right) \leq m-\frac{1}{6} \int_{\mathbb{R}^{3}} \beta(t, x) \bar{u}^{2} \mathrm{~d} x \\
& \leq m-\frac{\delta_{2}}{6}, \quad \forall t>0, \quad|t-1| \geq \delta_{1},
\end{aligned}
$$

where

$$
\delta_{2}:=\min \left\{\int_{\mathbb{R}^{3}} \beta\left(1-\delta_{1}, x\right) \bar{u}^{2} \mathrm{~d} x, \int_{\mathbb{R}^{3}} \beta\left(1+\delta_{1}, x\right) \bar{u}^{2} \mathrm{~d} x\right\}>0
$$


Combining (3.52) with (3.53), we have

$$
\max _{t \in\left[T_{1}, T_{2}\right]} \mathcal{I}\left(\eta\left(1, t^{2} \bar{u}_{t}\right)\right)<m
$$

Define the function $\Psi_{0}(t):=\mathcal{J}\left(\eta\left(1, t^{2} \bar{u}_{t}\right)\right)$ for all $t>0$. It follows from (3.51) and i) that $\eta\left(1, t^{2} \bar{u}_{t}\right)=t^{2} \bar{u}_{t}$ for $t=T_{1}$ and $t=T_{2}$, which, together with (3.50), implies

$$
\Psi_{0}\left(T_{1}\right)=\mathcal{J}\left(T_{1}^{2} \bar{u}_{T_{1}}\right)>0, \quad \Psi_{0}\left(T_{2}\right)=\mathcal{J}\left(T_{2}^{2} \bar{u}_{T_{2}}\right)<0 .
$$

Since $\Psi_{0}(t)$ is continuous on $(0, \infty)$, then we have that $\eta\left(1, t^{2} \bar{u}_{t}\right) \cap \mathcal{M} \neq \varnothing$ for some $t_{0} \in\left[T_{1}, T_{2}\right]$, contradicting the definition of $m$.

Proof of Theorem 1.4 In view of Lemmas 3.13 and 3.14, there exists $\bar{u} \in \mathcal{M}$ such that

$$
\mathcal{I}(\bar{u})=m=\inf _{u \in H^{1}\left(\mathbb{R}^{3}\right) \backslash\{0\}} \max _{t>0} \mathcal{I}\left(t^{2} u_{t}\right), \quad \mathcal{I}^{\prime}(\bar{u})=0 .
$$

This shows that $\bar{u}$ is a ground state solution of (1.1) such that $\mathcal{I}(\bar{u})=m=\inf _{\mathcal{M}} \mathcal{I}$.

Remark 3.15 As in the proof of Theorem 1.4, by replacing Lemma 3.4 with Lemma 3.7, we then obtain Corollary 1.6.

\section{Proof of Theorem 1.1}

In this section, we give the proof of Theorem 1.1. Without loss of generality, we consider that $V(x) \not \equiv V_{\infty}$.

Proposition 4.1 [21] Let X be a Banach space and let $J \subset \mathbb{R}^{+}$be an interval, and

$$
\Phi_{\lambda}(u)=A(u)-\lambda B(u), \quad \forall \lambda \in J,
$$

be a family of $\mathcal{C}^{1}$-functionals on $X$ such that

(i) either $A(u) \rightarrow+\infty$ or $B(u) \rightarrow+\infty$, as $\|u\| \rightarrow \infty$;

(ii) $B$ maps every bounded set of $X$ into a set of $\mathbb{R}$ bounded below;

(iii) there are two points $v_{1}, v_{2}$ in $X$ such that

$$
\tilde{c}_{\lambda}:=\inf _{\gamma \in \tilde{\Gamma}} \max _{t \in[0,1]} \Phi_{\lambda}(\gamma(t))>\max \left\{\Phi_{\lambda}\left(v_{1}\right), \Phi_{\lambda}\left(v_{2}\right)\right\}
$$

where

$$
\tilde{\Gamma}=\left\{\gamma \in \mathcal{C}([0,1], X): \gamma(0)=v_{1}, \gamma(1)=v_{2}\right\}
$$

Then, for almost every $\lambda \in J$, there exists a sequence $\left\{u_{n}(\lambda)\right\}$ such that

(i) $\left\{u_{n}(\lambda)\right\}$ is bounded in $X$; 
(ii) $\Phi_{\lambda}\left(u_{n}(\lambda)\right) \rightarrow \tilde{c}_{\lambda}$;

(iii) $\Phi_{\lambda}^{\prime}\left(u_{n}(\lambda)\right) \rightarrow 0$ in $X^{*}$, where $X^{*}$ is the dual of $X$.

For $\lambda \in[1 / 2,1]$ we introduce two families of $\mathcal{C}^{1}$-functionals on $H^{1}\left(\mathbb{R}^{3}\right)$ defined by

$$
\begin{aligned}
\mathcal{I}_{\lambda}(u) & :=\frac{1}{2} \int_{\mathbb{R}^{3}}\left(|\nabla u|^{2}+V(x) u^{2}\right) \mathrm{d} x+\frac{q^{2}}{4} \int_{\mathbb{R}^{3}} \phi_{u}(x) u^{2} \mathrm{~d} x-\lambda \int_{\mathbb{R}^{3}} F(u) \mathrm{d} x, \\
\mathcal{I}_{\lambda}^{\infty}(u) & :=\frac{1}{2} \int_{\mathbb{R}^{3}}\left(|\nabla u|^{2}+V_{\infty} u^{2}\right) \mathrm{d} x+\frac{q^{2}}{4} \int_{\mathbb{R}^{3}} \phi_{u}(x) u^{2} \mathrm{~d} x-\lambda \int_{\mathbb{R}^{3}} F(u) \mathrm{d} x .
\end{aligned}
$$

In view of [18, A.14], we obtain the following useful identity.

Lemma 4.2 Assume that (V1), (V2) and (F1)-(F3) hold. Let u be a critical point of $\mathcal{I}_{\lambda}$ in $H^{1}\left(\mathbb{R}^{3}\right)$, then the following Pohožaev-type identity holds

$$
\begin{aligned}
\mathcal{P}_{\lambda}(u):= & \frac{1}{2}\|\nabla u\|_{2}^{2}+\frac{1}{2} \int_{\mathbb{R}^{3}}[3 V(x)+\nabla V(x) \cdot x] u^{2} \mathrm{~d} x-3 \lambda \int_{\mathbb{R}^{3}} F(u) \mathrm{d} x \\
& +\frac{5 q^{2}}{4} \int_{\mathbb{R}^{3}} \phi_{u} u^{2} \mathrm{~d} x+\frac{q^{2}}{4 a} \int_{\mathbb{R}^{3}} \int_{\mathbb{R}^{3}} e^{-\frac{|x-y|}{a}} u^{2}(x) u^{2}(y) \mathrm{d} x \mathrm{~d} y=0 .
\end{aligned}
$$

Let us set $\mathcal{J}_{\lambda}(u):=2 \mathcal{I}_{\lambda}^{\prime}(u)[u]-\mathcal{P}_{\lambda}(u)$ for all $\lambda \in[1 / 2,1]$. Then

$$
\begin{aligned}
\mathcal{J}_{\lambda}(u)= & \frac{3}{2}\|\nabla u\|_{2}^{2}+\frac{1}{2} \int_{\mathbb{R}^{3}}[V(x)-\nabla V(x) \cdot x] u^{2} \mathrm{~d} x-\lambda \int_{\mathbb{R}^{3}}[2 f(u) u-3 F(u)] \mathrm{d} x \\
& +\frac{3 q^{2}}{4} \int_{\mathbb{R}^{3}} \phi_{u} u^{2} \mathrm{~d} x-\frac{q^{2}}{4 a} \int_{\mathbb{R}^{3}} \int_{\mathbb{R}^{3}} e^{-\frac{|x-y|}{a}} u^{2}(x) u^{2}(y) \mathrm{d} x \mathrm{~d} y .
\end{aligned}
$$

Similarly, for all $\lambda \in[1 / 2,1]$, if $u$ is a critical point of $\mathcal{I}_{\lambda}^{\infty}$, then $u$ satisfies the following Pohožaev-type identity:

$$
\begin{aligned}
\mathcal{P}_{\lambda}^{\infty}(u):= & \frac{1}{2}\|\nabla u\|_{2}^{2}+\frac{3 V_{\infty}}{2} \int_{\mathbb{R}^{3}}\|u\|_{2}^{2}-3 \lambda \int_{\mathbb{R}^{3}} F(u) \mathrm{d} x \\
& +\frac{5 q^{2}}{4} \int_{\mathbb{R}^{3}} \phi_{u} u^{2} \mathrm{~d} x+\frac{q^{2}}{4 a} \int_{\mathbb{R}^{3}} \int_{\mathbb{R}^{3}} e^{-\frac{|x-y|}{a}} u^{2}(x) u^{2}(y) \mathrm{d} x \mathrm{~d} y .=0
\end{aligned}
$$

We also let

$$
\begin{aligned}
\mathcal{J}_{\lambda}^{\infty}(u)= & \frac{3}{2}\|\nabla u\|_{2}^{2}+\frac{V_{\infty}}{2}\|u\|_{2}^{2}-\lambda \int_{\mathbb{R}^{3}}[2 f(u) u-3 F(u)] \mathrm{d} x \\
& +\frac{3 q^{2}}{4 a} \int_{\mathbb{R}^{3}} \phi_{u} u^{2} \mathrm{~d} x-\frac{q^{2}}{4 a} \int_{\mathbb{R}^{3}} \int_{\mathbb{R}^{3}} e^{-\frac{|x-y|}{a} u^{2}(x) u^{2}(y) \mathrm{d} x \mathrm{~d} y .}
\end{aligned}
$$


Define

$$
\mathcal{M}_{\lambda}^{\infty}:=\left\{u \in H^{1}\left(\mathbb{R}^{3}\right) \backslash\{0\}: \mathcal{J}_{\lambda}^{\infty}(u)=0\right\}, \quad m_{\lambda}^{\infty}:=\inf _{\mathcal{M}_{\lambda}^{\infty}} \mathcal{I}_{\lambda}^{\infty}
$$

By Lemma 3.7, we have the following lemma.

Lemma 4.3 Assume that (F1), (F3) and (F4) hold. Then

$$
\begin{aligned}
\mathcal{I}_{\lambda}^{\infty}(u) \geq & \mathcal{I}_{\lambda}^{\infty}\left(t^{2} u_{t}\right)+\frac{1-t^{3}}{3} \mathcal{J}_{\lambda}^{\infty}(u) \\
& +\frac{(1-t)^{2}(2+t)}{6} V_{\infty}\|u\|_{2}^{2}, \quad \forall u \in H^{1}\left(\mathbb{R}^{3}\right), t>0 .
\end{aligned}
$$

In view of Corollary $1.6, \mathcal{I}_{1}^{\infty}=\mathcal{I}^{\infty}$ has a minimizer $u_{1}^{\infty} \neq 0$ on $\mathcal{M}_{1}^{\infty}=\mathcal{M}^{\infty}$, i.e.

$$
u_{1}^{\infty} \in \mathcal{M}_{1}^{\infty}, \quad\left(\mathcal{I}_{1}^{\infty}\right)^{\prime}\left(u_{1}^{\infty}\right)=0 \quad \text { and } \quad m_{1}^{\infty}=\mathcal{I}_{1}^{\infty}\left(u_{1}^{\infty}\right)
$$

Noting that (1.5) is autonomous, $V \in \mathcal{C}\left(\mathbb{R}^{3}, \mathbb{R}\right)$ and $V(x) \leq V_{\infty}$ but $V(x) \not \equiv V_{\infty}$, we can find $\bar{x} \in \mathbb{R}^{3}$ and $\bar{r}>0$ such that

$$
V_{\infty}-V(x)>0, \quad\left|u_{1}^{\infty}(x)\right|>0 \quad \text { a.e. }|x-\bar{x}| \leq \bar{r}
$$

after suitable translations to $u_{1}^{\infty}$.

By (V1), we have $V_{\max }:=\max _{x \in \mathbb{R}^{3}} V(x) \in(0, \infty)$. Let

$$
\begin{aligned}
\mathcal{I}_{\lambda}^{*}(u)= & \frac{1}{2} \int_{\mathbb{R}^{3}}\left(|\nabla u|^{2}+V_{\max } u^{2}\right) \mathrm{d} x+\frac{q^{2}}{4} \int_{\mathbb{R}^{3}} \phi_{u}(x) u^{2} \mathrm{~d} x \\
& -\lambda \int_{\mathbb{R}^{3}} F(u) \mathrm{d} x .
\end{aligned}
$$

Then it follows from (3.19) and (4.10) that there exists $T>0$ such that

$$
\mathcal{I}_{1 / 2}^{*}\left(t^{2}\left(u_{1}^{\infty}\right)_{t}\right)<0, \quad \forall t \geq T
$$

Lemma 4.4 Assume that (V1), (V2) and (F1)-(F3) hold. Then

(i) there exists $T>0$, independent of $\lambda$, such that $\mathcal{I}_{\lambda}\left(T^{2}\left(u_{1}^{\infty}\right)_{T}\right)<0$ for all $\lambda \in[1 / 2,1]$

(ii) there exists a positive constant $\kappa_{0}$, independent of $\lambda$, such that for all $\lambda \in[1 / 2,1]$,

$$
c_{\lambda}:=\inf _{\gamma \in \Gamma} \max _{t \in[0,1]} \mathcal{I}_{\lambda}(\gamma(t)) \geq \kappa_{0}>\max \left\{\mathcal{I}_{\lambda}(0), \mathcal{I}_{\lambda}\left(T^{2}\left(u_{1}^{\infty}\right)_{T}\right)\right\}
$$

where

$$
\Gamma=\left\{\gamma \in \mathcal{C}\left([0,1], H^{1}\left(\mathbb{R}^{3}\right)\right): \gamma(0)=0, \gamma(1)=T^{2}\left(u_{1}^{\infty}\right)_{T}\right\}
$$


(iii) $c_{\lambda}$ is bounded for $\lambda \in[1 / 2,1]$ and $\lim \sup _{\lambda \rightarrow \lambda_{0}} c_{\lambda} \leq c_{\lambda_{0}}$ for all $\lambda_{0} \in(1 / 2,1]$;

(iv) if $f$ further satisfies (F4), then $m_{\lambda}^{\infty}$ are non-increasing on $\lambda \in[1 / 2,1]$.

The proof of Lemma 4.4 is standard, so we omit it. Moreover, similarly to proof of [15, Lemma 4.5], we have the following lemma.

Lemma 4.5 Assume that (V1), (V2) and (F1)-(F4) hold. Then there exists $\bar{\lambda} \in[1 / 2,1)$ such that $c_{\lambda}<m_{\lambda}^{\infty}$ for all $\lambda \in(\bar{\lambda}, 1]$.

Lemma 4.6 Assume that (V1), (V2) and (F1)-(F4) hold. Then for almost every $\lambda \in$ $(\bar{\lambda}, 1]$, there exists $u_{\lambda} \in H^{1}\left(\mathbb{R}^{3}\right) \backslash\{0\}$ such that

$$
\mathcal{I}_{\lambda}^{\prime}\left(u_{\lambda}\right)=0, \quad \mathcal{I}_{\lambda}\left(u_{\lambda}\right)=c_{\lambda}
$$

Proof By Proposition 4.1, for almost every $\lambda \in[1 / 2,1]$, there exists a bounded sequence $\left\{u_{n}(\lambda)\right\} \subset H^{1}\left(\mathbb{R}^{3}\right)$, which we denote it by $\left\{u_{n}\right\}$ for simplicity, such that

$$
\mathcal{I}_{\lambda}\left(u_{n}\right) \rightarrow c_{\lambda}>0, \quad \mathcal{I}_{\lambda}^{\prime}\left(u_{n}\right) \rightarrow 0
$$

Similarly to the proof of [18, Lemma 4.5], using Lemma 3.12, we then deduce that there exist $u_{\lambda} \in H^{1}\left(\mathbb{R}^{3}\right)$, an integer $l \in \mathbb{N} \cup\{0\}$, a sequence $\left\{y_{n}^{k}\right\} \subset \mathbb{R}^{3}$ and $w^{k} \in H^{1}\left(\mathbb{R}^{3}\right)$ for $1 \leq k \leq l$ such that $u_{n} \rightarrow u_{\lambda}$ in $H^{1}\left(\mathbb{R}^{3}\right), \mathcal{I}_{\lambda}^{\prime}\left(u_{\lambda}\right)=0,\left(\mathcal{I}_{\lambda}^{\infty}\right)^{\prime}\left(w^{k}\right)=0$ and $\mathcal{I}_{\lambda}^{\infty}\left(w^{\bar{k}}\right) \geq m_{\lambda}^{\infty}$ for $1 \leq k \leq l$,

$$
\left\|u_{n}-u_{\lambda}-\sum_{k=1}^{l} w^{k}\left(\cdot+y_{n}^{k}\right)\right\| \rightarrow 0 \text { and } \mathcal{I}_{\lambda}\left(u_{n}\right) \rightarrow \mathcal{I}_{\lambda}\left(u_{\lambda}\right)+\sum_{i=1}^{l} \mathcal{I}_{\lambda}^{\infty}\left(w^{i}\right) .
$$

Since $\mathcal{I}_{\lambda}^{\prime}\left(u_{\lambda}\right)=0$, then $\mathcal{J}_{\lambda}\left(u_{\lambda}\right)=0$. It follows from (V2), (3.6), (4.2) and (4.5) that

$$
\begin{aligned}
\mathcal{I}_{\lambda}\left(u_{\lambda}\right)= & \mathcal{I}_{\lambda}\left(u_{\lambda}\right)-\frac{1}{3} \mathcal{J}_{\lambda}\left(u_{\lambda}\right) \\
= & \frac{1}{6} \int_{\mathbb{R}^{3}}[2 V(x)+\nabla V(x) \cdot x] u_{\lambda}^{2} \mathrm{~d} x+\frac{q^{2}}{12 a} \int_{\mathbb{R}^{3}} \int_{\mathbb{R}^{3}} e^{-\frac{|x-y|}{a}} u^{2}(x) u^{2}(y) \mathrm{d} x \mathrm{~d} y \\
& +\frac{2 \lambda}{3} \int_{\mathbb{R}^{3}}\left[f\left(u_{\lambda}\right) u_{\lambda}-3 F\left(u_{\lambda}\right)\right] \mathrm{d} x \geq 0 .
\end{aligned}
$$

If $l \neq 0$, then

$$
c_{\lambda}=\lim _{n \rightarrow \infty} \mathcal{I}_{\lambda}\left(u_{n}\right)=\mathcal{I}_{\lambda}\left(u_{\lambda}\right)+\sum_{i=1}^{l} \mathcal{I}_{\lambda}^{\infty}\left(w^{i}\right) \geq m_{\lambda}^{\infty}, \quad \forall \lambda \in(\bar{\lambda}, 1],
$$

which contradicts Lemma 4.5. Thus, $l=0$, and (4.16) implies that $u_{n} \rightarrow u_{\lambda}$ in $H^{1}\left(\mathbb{R}^{3}\right)$ and $\mathcal{I}_{\lambda}\left(u_{\lambda}\right)=c_{\lambda}$ for almost every $\lambda \in(\bar{\lambda}, 1]$. 
Lemma 4.7 Assume that (V1), (V2) and (F1)-(F4) hold. Then there exists $\bar{u} \in$ $H^{1}\left(\mathbb{R}^{3}\right) \backslash\{0\}$ such that

$$
\mathcal{I}^{\prime}(\bar{u})=0, \quad 0<\mathcal{I}(\bar{u}) \leq c_{1} .
$$

Proof In view of Lemma 4.4 (ii) and (iii) and Lemma 4.6, there exist two sequences $\left\{\lambda_{n}\right\} \subset(\bar{\lambda}, 1]$ and $\left\{u_{\lambda_{n}}\right\} \subset H^{1}\left(\mathbb{R}^{3}\right)$, which we denoted it by $\left\{u_{n}\right\}$ for brevity, such that

$$
\lambda_{n} \rightarrow 1, \quad c_{\lambda_{n}} \rightarrow c_{*}>0, \quad \mathcal{I}_{\lambda_{n}}^{\prime}\left(u_{n}\right)=0, \quad \mathcal{I}_{\lambda_{n}}\left(u_{n}\right)=c_{\lambda_{n}}
$$

Now we assert that $\left\{u_{n}\right\}$ is bounded in $H^{1}\left(\mathbb{R}^{3}\right)$.

By (4.2), (4.5), (4.19) and Lemma 4.4 (iii), one has

$$
\begin{aligned}
C_{1} \geq & c_{\lambda_{n}}=\mathcal{I}_{\lambda_{n}}\left(u_{n}\right)-\frac{1}{3} \mathcal{J}_{\lambda_{n}}\left(u_{n}\right) \\
= & \frac{1}{6} \int_{\mathbb{R}^{3}}[2 V(x)+\nabla V(x) \cdot x] u_{n}^{2} \mathrm{~d} x+\frac{q^{2}}{12 a} \int_{\mathbb{R}^{3}} \int_{\mathbb{R}^{3}} e^{-\frac{|x-y|}{a}} u_{n}^{2}(x) u_{n}^{2}(y) \mathrm{d} x \mathrm{~d} y \\
& \quad+\frac{2 \lambda_{n}}{3} \int_{\mathbb{R}^{3}}\left[f\left(u_{n}\right) u_{n}-3 F\left(u_{n}\right)\right] \mathrm{d} x
\end{aligned}
$$

By (V2), there exist constants $\varrho_{0}, R_{0}>0$ such that

$$
2 V(x)+\nabla V(x) \cdot x \geq \varrho_{0}, \quad \forall|x| \geq R_{0} .
$$

Then it follows from (3.6), (4.20) and (4.21) that

$$
C_{1} \geq \frac{\varrho_{0}}{6} \int_{|x| \geq R_{0}} u_{n}^{2} \mathrm{~d} x+\frac{q^{2} e^{-\frac{2 R_{0}}{a}}}{12 a}\left(\int_{|x|<R_{0}} u_{n}^{2} \mathrm{~d} x\right)^{2}
$$

which implies that $\left\{\left\|u_{n}\right\|_{2}\right\}$ is bounded.

Next, we prove that $\left\{\left\|\nabla u_{n}\right\|_{2}\right\}$ is also bounded. Arguing by contradiction, suppose that $\left\|\nabla u_{n}\right\|_{2} \rightarrow \infty$. By (V1), (V2), (4.22) and Lemma 4.4 (iii), one has

$$
c_{\lambda_{n}}+\int_{\mathbb{R}^{3}}\left[V_{\infty}-V(x)+|\nabla V(x) \cdot x|\right] u_{n}^{2} \mathrm{~d} x \leq M_{0}
$$

for some constant $M_{0}>0$. Let $t_{n}=\min \left\{1,2\left(M_{0} /\left\|\nabla u_{n}\right\|_{2}^{2}\right)^{1 / 3}\right\}$. Then $t_{n} \rightarrow 0$. Thus, it follows from (4.2), (4.3), (4.5), (4.7), (4.9) and (4.23) that

$$
\begin{aligned}
\mathcal{I}_{\lambda_{n}}^{\infty}\left(t_{n}^{2}\left(u_{n}\right)_{t_{n}}\right) & \leq \mathcal{I}_{\lambda_{n}}^{\infty}\left(u_{n}\right)-\frac{1-t_{n}^{3}}{3} \mathcal{J}_{\lambda_{n}}^{\infty}\left(u_{n}\right) \\
& =\mathcal{I}_{\lambda_{n}}\left(u_{n}\right)+\frac{1}{2} \int_{\mathbb{R}^{3}}\left[V_{\infty}-V(x)\right] u_{n}^{2} \mathrm{~d} x
\end{aligned}
$$




$$
\begin{gathered}
\quad-\frac{1-t_{n}^{3}}{3}\left[\mathcal{J}_{\lambda_{n}}\left(u_{n}\right)+\frac{1}{2} \int_{\mathbb{R}^{3}}\left[V_{\infty}-V(x)+\nabla V(x) \cdot x\right] u_{n}^{2} \mathrm{~d} x\right] \\
\leq c_{\lambda_{n}}+\int_{\mathbb{R}^{3}}\left[V_{\infty}-V(x)+|\nabla V(x) \cdot x|\right] u_{n}^{2} \mathrm{~d} x \leq M_{0}
\end{gathered}
$$

As in the proof of (3.39), we then deduce a contradiction by using (4.24). Hence, $\left\{u_{n}\right\}$ is bounded in $H^{1}\left(\mathbb{R}^{3}\right)$, and the assertion holds.

Similarly to the proof of Lemma 4.6, there exists $\bar{u} \in H^{1}\left(\mathbb{R}^{3}\right) \backslash\{0\}$ such that (4.18) holds.

Proof of Theorems 1.1 Define

$$
\mathcal{K}:=\left\{u \in H^{1}\left(\mathbb{R}^{3}\right) \backslash\{0\}: \mathcal{I}^{\prime}(u)=0\right\}, \quad \hat{m}:=\inf _{u \in \mathcal{K}} \mathcal{I}(u)
$$

Then Lemma 4.7 shows that $\mathcal{K} \neq \varnothing$ and $\hat{m} \leq c_{1}$. For any $u \in \mathcal{K},(3.16),(4.5)$ and Lemma 4.2 imply $\mathcal{J}(u)=\mathcal{J}_{1}(u)=2 \mathcal{I}^{\prime}(u)[u]-\mathcal{P}(u)=0$. By (2.5), (3.16) and (4.21), one has

$\mathcal{I}(u)=\mathcal{I}(u)-\frac{1}{3} \mathcal{J}(u) \geq \frac{\varrho_{0}}{6} \int_{|x| \geq R_{0}} u^{2} \mathrm{~d} x+\frac{q^{2} e^{-\frac{2 R_{0}}{a}}}{12 a}\left(\int_{|x|<R_{0}} u^{2} \mathrm{~d} x\right)^{2}>0, \quad \forall u \in \mathcal{K}$,

which implies $\hat{m} \geq 0$. Since $\mathcal{I}^{\prime}(u)[u]=0$ for $u \in \mathcal{K}$, we then deduce from (F1), (F2) and the Sobolev embedding theorem that there exists $\alpha_{0}>0$ such that

$$
\|u\| \geq \alpha_{0}, \quad \forall u \in \mathcal{K} .
$$

Let $\left\{u_{n}\right\} \subset \mathcal{K}$ be such that $\mathcal{I}^{\prime}\left(u_{n}\right)=0$ and $\mathcal{I}\left(u_{n}\right) \rightarrow \hat{m}$. In view of Lemma 4.5, we have $\hat{m} \leq c_{1}<m_{1}^{\infty}$. Similarly to the proof of Lemma 4.6, we deduce that there exists $\hat{u} \in H^{1}\left(\mathbb{R}^{3}\right)$ such that $u_{n} \rightarrow \hat{u}$ in $H^{1}\left(\mathbb{R}^{3}\right), \mathcal{I}^{\prime}(\hat{u})=0$ and $\mathcal{I}(\hat{u})=\hat{m}$. Moreover, (4.25) leads to $\hat{u} \neq 0$. Hence, $\hat{u} \in H^{1}\left(\mathbb{R}^{3}\right)$ is a ground state solution of (1.1).

Proof of Theorems 1.3 As in the proof of Lemma 4.6, for almost every $\lambda \in[1 / 2,1]$, there exists a bounded sequence $\left\{u_{n}(\lambda)\right\} \subset H^{1}\left(\mathbb{R}^{3}\right)$, which we denote it by $\left\{u_{n}\right\}$ for simplicity, and a positive constant $\kappa_{0}^{\infty}$, independent of $\lambda$, such that

$$
\mathcal{I}_{\lambda}^{\infty}\left(u_{n}\right) \rightarrow c_{\lambda}^{\infty} \geq \kappa_{0}^{\infty}, \quad\left(\mathcal{I}_{\lambda}^{\infty}\right)^{\prime}\left(u_{n}\right) \rightarrow 0 .
$$

Using (F1), (F2), (4.26) and [26, Lemma 1.21], we can prove that there exists a sequence $y_{n} \in \mathbb{R}^{3}$ such that $\int_{B_{1}\left(y_{n}\right)}\left|u_{n}\right|^{2} \mathrm{~d} x>0$. Let $\bar{u}_{n}(x)=u_{n}\left(x+y_{n}\right)$. Then $\left\|\bar{u}_{n}\right\|=\left\|u_{n}\right\|$ and there exists $\bar{u} \in H^{1}\left(\mathbb{R}^{3}\right) \backslash\{0\}$ such that $\bar{u}_{n} \rightarrow \tilde{u}$ in $H^{1}\left(\mathbb{R}^{3}\right)$. Note that

$$
\mathcal{I}_{\lambda}^{\infty}\left(\bar{u}_{n}\right) \rightarrow c_{\lambda}^{\infty} \geq \kappa_{0}^{\infty}, \quad\left(\mathcal{I}_{\lambda}^{\infty}\right)^{\prime}\left(\bar{u}_{n}\right) \rightarrow 0
$$

By a standard argument, for almost every $\lambda \in[1 / 2,1]$, there exists $u_{\lambda} \in H^{1}\left(\mathbb{R}^{3}\right) \backslash\{0\}$ such that

$$
\left(\mathcal{I}_{\lambda}^{\infty}\right)^{\prime}\left(u_{\lambda}\right)=0, \quad \mathcal{I}_{\lambda}^{\infty}\left(u_{\lambda}\right)=c_{\lambda}^{\infty} \geq \kappa_{0}^{\infty}
$$


From (4.28), there exist two sequences $\left\{\lambda_{n}\right\} \subset[1 / 2,1]$ and $\left\{u_{\lambda_{n}}\right\} \subset H^{1}\left(\mathbb{R}^{3}\right)$, which we denote the latter by $\left\{u_{n}\right\}$, such that

$$
\lambda_{n} \rightarrow 1, \quad \kappa_{0}^{\infty} \leq c_{\lambda_{n}}^{\infty} \rightarrow c^{\infty}, \quad\left(\mathcal{I}_{\lambda_{n}}^{\infty}\right)^{\prime}\left(u_{n}\right)=0, \quad \mathcal{I}_{\lambda_{n}}^{\infty}\left(u_{n}\right)=c_{\lambda_{n}}^{\infty}
$$

Similarly to (4.20), we have

$$
\begin{aligned}
C_{2} \geq & c_{\lambda_{n}}^{\infty}=\mathcal{I}_{\lambda_{n}}^{\infty}\left(u_{n}\right)-\frac{1}{3} \mathcal{J}_{\lambda_{n}}^{\infty}\left(u_{n}\right) \\
= & \frac{V_{\infty}}{3}\left\|u_{n}\right\|_{2}^{2}+\frac{q^{2}}{12 a} \int_{\mathbb{R}^{3}} \int_{\mathbb{R}^{3}} e^{-\frac{|x-y|}{a}} u_{n}^{2}(x) u_{n}^{2}(y) \mathrm{d} x \mathrm{~d} y \\
& \quad+\frac{2 \lambda_{n}}{3} \int_{\mathbb{R}^{3}}\left[f\left(u_{n}\right) u_{n}-3 F\left(u_{n}\right)\right] \mathrm{d} x,
\end{aligned}
$$

which implies

$$
\left\|u_{n}\right\|_{2}^{2} \leq C_{3}, \quad \int_{\mathbb{R}^{3}}\left[f\left(u_{n}\right) u_{n}-3 F\left(u_{n}\right)\right] \mathrm{d} x \leq C_{4},
$$

and

$$
\int_{\mathbb{R}^{3}} \int_{\mathbb{R}^{3}} e^{-\frac{|x-y|}{a}} u_{n}^{2}(x) u_{n}^{2}(y) \mathrm{d} x \mathrm{~d} y \leq C_{5},
$$

Next, we claim that $\left\{\left\|\nabla u_{n}\right\|_{2}\right\}$ is also bounded. Arguing by contradiction, suppose that $\left\|\nabla u_{n}\right\|_{2} \rightarrow \infty$. Set $v_{n}=u_{n} /\left\|u_{n}\right\|$, then $\left\|v_{n}\right\|=1$, and (4.31) implies $\left\|v_{n}\right\|_{2} \rightarrow 0$.

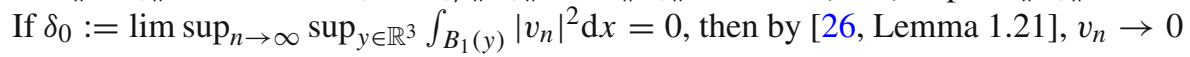
in $L^{s}\left(\mathbb{R}^{3}\right)$ for $2<s<6$.

Since $\left\|v_{n}\right\|_{2} \rightarrow 0$, we have

$$
\int_{0<\left|u_{n}\right| \leq r_{0}} \frac{f\left(u_{n}\right)}{u_{n}} v_{n}^{2} \mathrm{~d} x \leq C_{6}\left\|v_{n}\right\|_{2}^{2}=o(1) .
$$

Set $\kappa^{\prime}=\kappa /(\kappa-1)$. Then (F5), (4.31) and the Hölder inequality yield

$$
\begin{aligned}
\int_{\left|u_{n}\right|>r_{0}} \frac{f\left(u_{n}\right)}{u_{n}} v_{n}^{2} \mathrm{~d} x & \leq\left[\int_{\left|u_{n}\right|>r_{0}}\left|\frac{f\left(u_{n}\right)}{u_{n}}\right|^{\kappa} \mathrm{d} x\right]^{1 / \kappa}\left\|v_{n}\right\|_{2 \kappa^{\prime}}^{2} \\
& \leq C_{7}\left(\int_{\left|u_{n}\right|>r_{0}}\left[f\left(u_{n}\right) u_{n}-3 F\left(u_{n}\right)\right] \mathrm{d} x\right)^{1 / \kappa}\left\|v_{n}\right\|_{2 \kappa^{\prime}}^{2} \\
& \leq C_{8}\left\|v_{n}\right\|_{2 \kappa^{\prime}}^{2}=o(1)
\end{aligned}
$$

Since $\left(\mathcal{I}_{\lambda_{n}}^{\infty}\right)^{\prime}\left(u_{n}\right)\left[u_{n}\right]=0$ by (4.29), then (4.33) and (4.34) yield 


$$
\begin{aligned}
1 & \leq \frac{1}{\left\|u_{n}\right\|^{2}}\left[\int_{\mathbb{R}^{3}}\left(\left|\nabla u_{n}\right|^{2}+V_{\infty} u_{n}^{2}\right) \mathrm{d} x+q^{2} \int_{\mathbb{R}^{3}} \phi_{u_{n}}(x) u_{n}^{2} \mathrm{~d} x\right] \\
& =\lambda_{n} \int_{\mathbb{R}^{3}} \frac{f\left(u_{n}\right)}{u_{n}} v_{n}^{2} \mathrm{~d} x=o(1) .
\end{aligned}
$$

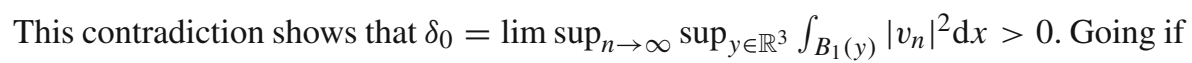
necessary to a subsequence, we may assume that there exists a sequence $\left\{y_{n}\right\} \subset \mathbb{R}^{3}$ such that $\int_{B_{1}\left(y_{n}\right)}\left|v_{n}\right|^{2} \mathrm{~d} x>\frac{\delta_{0}}{2}$ for all $n \in \mathbb{N}$. Let $w_{n}(x)=v_{n}\left(x+y_{n}\right)$. Then $\left\|w_{n}\right\|=$ $\left\|v_{n}\right\|=1$, and for all $n \in \mathbb{N}$

$$
\int_{B_{1}(0)}\left|w_{n}\right|^{2} \mathrm{~d} x>\frac{\delta_{0}}{2} .
$$

Then there exists $w \in H^{1}\left(\mathbb{R}^{3}\right) \backslash\{0\}$ such that, passing to a subsequence, $w_{n} \rightarrow w$ in $H^{1}\left(\mathbb{R}^{3}\right), w_{n} \rightarrow w$ in $L_{\text {loc }}^{s}\left(\mathbb{R}^{3}\right)$ for all $1 \leq s<6, w_{n} \rightarrow w$ a.e. in $\mathbb{R}^{3}$. Let us define $\tilde{u}_{n}(x)=u_{n}\left(x+y_{n}\right)$. Then $\tilde{u}_{n} /\left\|u_{n}\right\|=w_{n} \rightarrow w$ a.e. in $\mathbb{R}^{3}$ and $w \neq 0$. For $x \in\left\{y \in \mathbb{R}^{3}: w(y) \neq 0\right\}$, we have $\lim _{n \rightarrow \infty}\left|\tilde{u}_{n}(x)\right|=\infty$. By (F1) and (F2), there exists $M_{1}>0$ such that

$$
F(t)+M_{1} t^{2} \geq 0, \quad \forall t \in \mathbb{R} .
$$

Note that (4.29) and (4.32) lead to

$$
\lambda_{n} \rightarrow 1, \quad \kappa_{0}^{\infty} \leq c_{\lambda_{n}}^{\infty} \rightarrow c^{\infty}, \quad\left(\mathcal{I}_{\lambda_{n}}^{\infty}\right)^{\prime}\left(\tilde{u}_{n}\right)=0, \quad \mathcal{I}_{\lambda_{n}}^{\infty}\left(\tilde{u}_{n}\right)=c_{\lambda_{n}}^{\infty}
$$

and

$$
\int_{\mathbb{R}^{3}} \int_{\mathbb{R}^{3}} e^{-\frac{|x-y|}{a}} \tilde{u}_{n}^{2}(x) \tilde{u}_{n}^{2}(y) \mathrm{d} x \mathrm{~d} y \leq C_{5} .
$$

From (F3), (4.3), (4.6), (4.37), (4.38), Lemma 4.2 and Fatou's lemma, we derive

$$
\begin{aligned}
0= & \lim _{n \rightarrow \infty} \frac{\mathcal{I}_{\lambda}^{\infty}\left(\tilde{u}_{n}\right)-\frac{1}{5} \mathcal{P}_{\lambda}^{\infty}\left(\tilde{u}_{n}\right)}{\left\|\tilde{u}_{n}(x)\right\|^{3}} \\
= & \lim _{n \rightarrow \infty}\left\{\frac { 1 } { 5 \| \tilde { u } _ { n } ( x ) \| ^ { 3 } } \left[2\left\|\nabla \tilde{u}_{n}\right\|_{2}^{2}+V_{\infty}\left\|\tilde{u}_{n}\right\|_{2}^{2}-\frac{q^{2}}{4 a} \int_{\mathbb{R}^{3}} \int_{\mathbb{R}^{3}} e^{\left.-\frac{|x-y|}{a} \tilde{u}^{2}(x) \tilde{u}^{2}(y) \mathrm{d} x \mathrm{~d} y\right]}\right.\right. \\
& \left.-\frac{2 \lambda_{n}}{5\left\|u_{n}\right\|^{3}} \int_{\mathbb{R}^{3}} F\left(\tilde{u}_{n}\right) \mathrm{d} x\right\} \\
\leq & -\frac{1}{5} \liminf _{n \rightarrow \infty} \int_{\mathbb{R}^{3}} \frac{F\left(\tilde{u}_{n}\right)+M_{1} \tilde{u}_{n}^{2}}{\left|\tilde{u}_{n}\right|^{3}} w_{n}^{3} \mathrm{~d} x=-\infty
\end{aligned}
$$

This contradiction shows that $\left\{u_{n}\right\}$ is bounded in $H^{1}\left(\mathbb{R}^{3}\right)$ and the claim holds.

As in the proof of Lemma 4.6, there exists $\bar{u} \in H^{1}\left(\mathbb{R}^{3}\right) \backslash\{0\}$ such that

$$
\left(\mathcal{I}^{\infty}\right)^{\prime}(\bar{u})=0, \quad 0<\mathcal{I}^{\infty}(\bar{u}) \leq c_{1}^{\infty}
$$


Set

$$
\mathcal{K}^{\infty}:=\left\{u \in H^{1}\left(\mathbb{R}^{3}\right) \backslash\{0\}:\left(\mathcal{I}^{\infty}\right)^{\prime}(u)=0\right\}, \quad \hat{m}^{\infty}:=\inf _{u \in \mathcal{K}^{\infty}} \mathcal{I}^{\infty}(u)
$$

The above argument shows that $\mathcal{K}^{\infty} \neq \varnothing$.

For any $u \in \mathcal{K}^{\infty}$, Lemma 4.2 implies $\mathcal{J}^{\infty}(u)=2\left(\mathcal{I}^{\infty}\right)^{\prime}(u)[u]-\mathcal{P}^{\infty}(u)=0$. By (F5) and (3.45), we have

$$
\mathcal{I}^{\infty}(u)=\mathcal{I}^{\infty}(u)-\frac{1}{3} \mathcal{J}^{\infty}(u) \geq \frac{V_{\infty}}{3}\|u\|_{2}^{2}>0, \quad \forall u \in \mathcal{K}^{\infty},
$$

which implies $\hat{m}^{\infty} \geq 0$. Since $\left(\mathcal{I}^{\infty}\right)^{\prime}(u)[u]=0$ for $u \in \mathcal{K}^{\infty}$, we easily deduce from (F1), (F2) and the Sobolev embedding theorem that there exists $\alpha_{\infty}>0$ such that

$$
\|u\| \geq \alpha_{\infty}, \quad \forall u \in \mathcal{K}^{\infty} .
$$

Let $\left\{u_{n}\right\} \subset \mathcal{K}^{\infty}$ be such that $\left(\mathcal{I}^{\infty}\right)^{\prime}\left(u_{n}\right)=0$ and $\mathcal{I}^{\infty}\left(u_{n}\right) \rightarrow \hat{m}^{\infty}$. Since $\left(\mathcal{I}^{\infty}\right)^{\prime}\left(u_{n}\right)\left[u_{n}\right]=0$, we can deduce from (4.39) and [26, Lemma 1.21] that $\left\{u_{n}\right\}$ is non-vanishing, and so up to a subsequence, there exists a sequence $\left\{y_{n}\right\} \subset \mathbb{R}^{3}$ such that $\int_{B_{1}\left(y_{n}\right)}\left|u_{n}\right|^{2} \mathrm{~d} x>0$. Let $\hat{u}_{n}(x)=v_{n}\left(x+y_{n}\right)$. Then there exists $\hat{u} \in H^{1}\left(\mathbb{R}^{3}\right) \backslash\{0\}$ such that $u_{n} \rightarrow \hat{u}$ in $H^{1}\left(\mathbb{R}^{3}\right),\left(\mathcal{I}^{\infty}\right)^{\prime}(\hat{u})=0$ and $\mathcal{I}^{\infty}(\hat{u}) \geq \hat{m}^{\infty}$. Moreover, it follows from (F5), (3.21), (3.22) and Fatou's lemma that

$$
\begin{aligned}
\hat{m}^{\infty}= & \lim _{n \rightarrow \infty}\left[\mathcal{I}^{\infty}\left(\hat{u}_{n}\right)-\frac{1}{3} \mathcal{J}^{\infty}\left(\hat{u}_{n}\right)\right] \\
= & \lim _{n \rightarrow \infty}\left[\frac{V_{\infty}}{3}\left\|\hat{u}_{n}\right\|_{2}^{2}+\frac{q^{2}}{12 a} \int_{\mathbb{R}^{3}} \int_{\mathbb{R}^{3}} e^{-\frac{|x-y|}{a}} \hat{u}_{n}^{2}(x) \hat{u}_{n}^{2}(y) \mathrm{d} x \mathrm{~d} y\right. \\
& \left.+\frac{2}{3} \int_{\mathbb{R}^{3}}\left[f\left(\hat{u}_{n}\right) \hat{u}_{n}-3 F\left(\hat{u}_{n}\right)\right] \mathrm{d} x\right] \\
\geq & \frac{V_{\infty}}{3}\|\hat{u}\|_{2}^{2}+\frac{q^{2}}{12 a} \int_{\mathbb{R}^{3}} \int_{\mathbb{R}^{3}} e^{-\frac{|x-y|}{a}} \hat{u}^{2}(x) \hat{u}^{2}(y) \mathrm{d} x \mathrm{~d} y \\
& +\frac{2}{3} \int_{\mathbb{R}^{3}}[f(\hat{u}) \hat{u}-3 F(\hat{u})] \mathrm{d} x \\
= & \mathcal{I}^{\infty}(\hat{u})-\frac{1}{3} \mathcal{J}^{\infty}(\hat{u})=\mathcal{I}^{\infty}(\hat{u}) \geq \hat{m}^{\infty},
\end{aligned}
$$

which implies $\mathcal{I}^{\infty}(\hat{u})=\hat{m}^{\infty}$. Hence, $\hat{u} \in H^{1}\left(\mathbb{R}^{3}\right)$ is a ground state solution of problem (1.5). The proof is now complete.

Author Contributions All authors contributed to the study conception and design. Material preparation, data collection and analysis were performed by all authors. The first draft of the manuscript was written by Sitong Chen and all authors commented on previous versions of the manuscript. The authors read and approved the final manuscript. 
Funding Sitong Chen is supported by the National Natural Science Foundation of China (No. 12001542), Lin Li is supported by the National Natural Science Foundation of China (No. 11601046), Chongqing Science and Technology Commission (No. cstc2016jcyjA0310). Xianhua Tang is supported by the National Natural Science Foundation of China (No. 11971485). The research of Vicenţiu D. Rădulescu was supported by a grant of the Romanian Ministry of Research, Innovation and Digitization, CNCS/CCCDI-UEFISCDI, project number PCE 137/2021, within PNCDI III.

Availability of data and materials All data generated or analysed during this study are included in this article.

\section{Declarations}

Conflict of interest The authors declare that they have no conflict of interest.

Open Access This article is licensed under a Creative Commons Attribution 4.0 International License, which permits use, sharing, adaptation, distribution and reproduction in any medium or format, as long as you give appropriate credit to the original author(s) and the source, provide a link to the Creative Commons licence, and indicate if changes were made. The images or other third party material in this article are included in the article's Creative Commons licence, unless indicated otherwise in a credit line to the material. If material is not included in the article's Creative Commons licence and your intended use is not permitted by statutory regulation or exceeds the permitted use, you will need to obtain permission directly from the copyright holder. To view a copy of this licence, visit http://creativecommons.org/licenses/by/4.0/.

\section{References}

1. Bertin, M.C., Pimentel, B.M., Valcárcel, C.E., Zambrano, G.E.R.: Hamilton-Jacobi formalism for Podolsky's electromagnetic theory on the null-plane. J. Math. Phys. 58, 082902 (2017)

2. Bonheure, D., Casteras, J.-B., dos Santos, E.M., Nascimento, R.: Orbitally stable standing waves of a mixed dispersion nonlinear Schrödinger equation. SIAM J. Math. Anal. 50, 5027-5071 (2018)

3. Bopp, F.: Eine Lineare theorie des Elektrons. Ann. Phys. 430, 345-384 (1940)

4. Born, M.: Modified field equations with a finite radius of the electron. Nature 132, 282 (1933)

5. Born, M.: On the quantum theory of the electromagnetic field. Proc. R. Soc. Lond. Ser. A 143, 410-437 (1934)

6. Born, M., Infeld, L.: Foundations of the new field theory. Nature 132, 1004 (1933)

7. Born, M., Infeld, L.: Foundations of the new field theory. Proc. R. Soc. Lond. Ser. A 144, 425-451 (1934)

8. Brezis, H., Lieb, E.: A relation between pointwise convergence of functions and convergence of functionals. Proc. Am. Math. Soc. 88, 486-490 (1983)

9. Bufalo, R., Pimentel, B.M., Soto, D.E.: Causal approach for the electron-positron scattering in generalized quantum electrodynamics. Phys. Rev. D 90, 085012 (2014)

10. Bufalo, R., Pimentel, B.M., Soto, D.E.: Normalizability analysis of the generalized quantum electrodynamics from the causal point of view. Int. J. Mod. Phys. A 32, 1750165 (2017)

11. Chen, S.T., Tang, X.H.: Berestycki-Lions conditions on ground state solutions for a nonlinear Schrödinger equation with variable potentials. Adv. Nonlinear Anal. 9(1), 496-515 (2020)

12. Chen, S.T., Tang, X.H.: On the planar Schrödinger-Poisson system with the axially symmetric potentials. J. Differ. Equ. 268, 945-976 (2020)

13. Chen, S.T., Fiscella, A., Pucci, P., Tang, X.H.: Semiclassical ground state solutions for critical Schrödinger-Poisson systems with lower perturbations. J. Differ. Equ. 268, 2672-2716 (2020)

14. Chen, S.T., Tang, X.H.: Axially symmetric solutions for the planar Schrödinger-Poisson system with critical exponential growth. J. Differ. Equ. 269, 9144-9174 (2020)

15. Chen, S.T., Tang, X.H.: On the critical Schrödinger-Bopp-Podolsky system with general nonlinearities. Nonlinear Anal. 195, 111734 (2020)

16. Cuzinatto, R.R., de Melo, C.A.M., Medeiros, L.G., Pimentel, B.M., Pompeia, P.J.: Bopp-Podolsky black holes and the no-hair theorem. Eur. Phys. Journal C 78, 43 (2018) 
17. Cuzinatto, R.R., de Melo, E.M., Medeiros, L.G., Souza, C.N.D., Pimentel, B.M.: De Broglie-Proca and Bopp-Podolsky massive photon gases in cosmology. Europhys. Lett. EPL 118, 19001 (2017)

18. d'Avenia, P., Siciliano, G.: Nonlinear Schrödinger equation in the Bopp-Podolsky electrodynamics: solutions in the electrostatic case. J. Differ. Equ. 267, 1025-1065 (2019)

19. Fibich, G., Ilan, B., Papanicolaou, G.: Self-focusing with fourth-order dispersion. SIAM J. Appl. Math. 62, 1437-1462 (2002)

20. Frenkel, J.: 4/3 problem in classical electrodynamics. Phys. Rev. E 54, 5859-5862 (1996)

21. Jeanjean, L.: On the existence of bounded Palais-Smale sequences and application to a LandesmanLazer-type problem set on $R^{N}$. Proc. R. Soc. Edinb. Sect. A 129, 787-809 (1999)

22. Mie, G.: Grundlagen einer Theorie der Materie. Ann. Phys. 345, 1-66 (1913)

23. Papageorgiou, N.S., Rădulescu, V.D., Repovš, D.: Nonlinear Analysis-Theory and Methods, Springer Monographs in Mathematics. Springer, Cham (2019)

24. Podolsky, B.: A generalized electrodynamics. Phys. Rev. 62, 68-71 (1942)

25. Tang, X.H., Chen, S.T.: Singularly perturbed Choquard equations with nonlinearity satisfying Berestycki-Lions assumptions. Adv. Nonlinear Anal. 9(1), 413-437 (2020)

26. Willem, M.: Minimax Theorems, Progress in Nonlinear Differential Equations and Their Applications, vol. 24. Birkhäuser Boston Inc., Boston (1996)

Publisher's Note Springer Nature remains neutral with regard to jurisdictional claims in published maps and institutional affiliations. 